\title{
GABAergic Neurons in the Rat Hippocampal Formation: Ultrastructure and Synaptic Relationships with Catecholaminergic Terminals
}

\author{
Teresa A. Milner and Catherine E. Bacon \\ Department of Neurology and Neurosciences, Division of Neurobiology, Cornell University Medical College, New York, New \\ York 10021
}

Numerous studies indicate that $\gamma$-aminobutyric acid (GABA) can either hyperpolarize or depolarize hippocampal pyramidal and granule cells. While the inhibitory action of GABA may occur directly on these cells, the excitatory action may be mediated by interactions of GABAergic neurons with each other or with catecholaminergic afferents. We sought to examine the cellular basis for these interactions and their relative frequency. Thus, the ultrastructural morphology of GABAergic neurons and their relation to terminals exhibiting immunoreactivity for the catecholamine-synthesizing enzyme tyrosine hydroxylase (TH) were examined in the rat hippocampal formation using combined immunoautoradiographic and peroxidase-antiperoxidase labeling methods. By light microscopy, GABAergic perikarya and processes codistributed most noticeably with TH-containing processes in the hilus of the dentate gyrus (DG) and in strata lucidum, radiatum, and lacunosum-moleculare of the CA3 region of the hippocampus. Thus, these regions were examined further by electron microscopy.

In the ultrastructural analysis, GABA-like immunoreactivity (GABA-LI) was detected in neuronal perikarya, dendrites, axons, and axon terminals. The GABA-containing perikarya were large, ovoid (20-40 $\mu \mathrm{m}$ in diameter), and contained abundant cytoplasm and an indented nucleus with one nucleolus. Synaptic junctions on the perikarya and dendrites with GABA-LI were both symmetric and asymmetric. Approximately equal numbers of TH-labeled terminals $(19 \%$ of 133 in DG; $39 \%$ of 26 in CA3) and GABA-containing terminals (19\% DG, 15\% CA3) formed synapses with GABA-labeled perikarya. The remainder of the presynaptic terminals $(62 \%$ DG, $46 \%$ CA3) were unlabeled, i.e., contained unidentified transmitters.

Terminals with GABA-LI $(0.5-1.6 \mu \mathrm{m})$ contained numerous small clear vesicles and from 0 to 2 large dense-core vesicles. The types of associations formed by terminals with GABA-LI were remarkably similar in the DG and hippocampus proper despite differences in intrinsic cell type and function. Terminals with GABA-LI formed associations with un-

\footnotetext{
Received Jan. 16, 1989; revised Mar. 6, 1989; accepted Mar. 9, 1989.

We would like to thank Drs. Chiye Aoki and Virginia M. Pickel for helpful suggestions on the manuscript and Drs. C. A bate and A. Towle for antisera against $\mathrm{TH}$ and GABA, respectively. Supported by NIMH grant MH42834 and NIH grant 18974.

Correspondence should be addressed to Dr. Teresa A. Milner, Department of Neurology and Neurosciences, Division of Neurobiology, Cornell University Medical College, 411 East 69th Street, New York, NY 10021.

Copyright (C) 1989 Society for Neuroscience $0270-6474 / 89 / 103410-18 \$ 02.00 / 0$
}

labeled perikarya and dendrites (24\% of 151 in DG, $25 \%$ of 75 in CA3) and synapses with GABA-containing perikarya and dendrites ( $18 \%$ DG, $5 \%$ CA3). Additionally, GABAergic terminals converged upon the same perikarya or dendrite as a TH-containing terminal (15\% DG, $21 \% \mathrm{CA} 3)$ and were in direct apposition to TH-labeled terminals (19\% DG, $20 \%$ CA3). The remaining GABAergic terminals (24\% DG, $28 \%$ CA3) were without any apparent synaptic relations. In both the DG and CA3, the junctions formed by GABAergic terminals were symmetric. Terminals showing colocalization of GABA-LI and TH-I were also detected, although rarely. These results provide cellular substrates in the hippocampal formation for direct synaptic modulation of (1) GABAergic neurons by both GABA and catecholamines and (2) pyramidal and granule cells as well as interneurons by GABAergic terminals either alone or in conjunction with catecholaminergic terminals. Both types of observed interactions can mediate the disinhibition (excitation) of hippocampal pyramidal and dentate granule cells.

Numerous physiological and pharmacological studies indicate that $\gamma$-aminobutyric acid (GABA) can either depolarize or hyperpolarize hippocampal pyramidal and granule cells (Curtis et al., 1970; Anderson et al., 1980; Jahnsen and Laursen, 1981; Alger and Nicoll, 1982a, b; Alger et al., 1982). The inhibition of pyramidal or granule cells by GABA is most likely a result of direct synaptic contacts from GABAergic terminals that arise from the GABAergic "basket" interneurons (Ribak et al., 1978; Kosaka et al., 1984; Gamrani et al., 1986). Some of the excitatory (i.e., disinhibitory) effects of GABA on hippocampal neurons are also due to inhibition of GABAergic interneurons by GABAergic terminals (Misgeld and Frotscher, 1986). However, the relative percentage of GABAergic terminals that synapse on GABAergic neurons versus non-GABAergic neurons has not been extensively investigated.

Recent studies also suggest that norepinephrine can also disinhibit hippocampal neurons either by decreasing the release of GABA from terminals or through direct contacts on GABAergic interneurons (Winson and Dahl, 1985; Madison and Nicoll, 1988). The possibility for such interactions is supported by the light microscopic detection of GABA-containing neurons and processes in an overlapping distribution with catecholaminergic terminals in the hippocampal formation (Sloviter and Nilaver, 1987; Milner and Bacon, 1989). Moreover, electron microscopic evidence in the CA3 region of the hippocampus (Frotscher and Leranth, 1988) indicates that catecholaminergic terminals synapse on neurons immunoreactive for glutamic acid decarbox- 
ylase (GAD), the synthetic enzyme for GABA. IIowever, the detection of GAD immunoreactivity in cell bodies requires colchicine pretreatment that could result in variable visualization of GABAergic neurons and thus would not be optimal for quantitative analysis. Moreover, whether similar synaptic relations occur in the dentate gyrus (DG) has not been determined. In addition, whether GABAergic terminals can interact directly with catecholaminergic terminals or indirectly through a common target in either the hippocampus or DG is not known.

Thus, we examined the cellular basis for interactions of GABAergic neurons with catecholaminergic terminals in the hippocampal formation using dual labeling methods (Pickel et al., 1986) for the detection of a rat polyclonal antibody against GABA and a rabbit antiserum against the general catecholamine-synthesizing enzyme, tyrosine hydroxylase (TH), in single sections. We sought to determine: (1) the topographic distribution of GABA-immunoreactive neurons in relation to $\mathrm{TH}$-containing processes and (2) the synaptic relations between neurons and terminals with GABA-like immunoreactivity (GABA-LI) and terminals immunoreactive for TH. We demonstrate direct synaptic relationships between catecholaminergic terminals and GABAergic neurons. Moreover, we show that GABAergic and catecholaminergic terminals are in direct apposition to each other and sometimes synapse on common targets. Preliminary results of this study have been reported previously in abstract form (Bacon et al., 1988).

\section{Materials and Methods}

Fixation and preparation of sections for immunocytochemistry. Studies were conducted in 6 adult male Sprague-Dawley rats (225-275 gm; Hilltop Lab. Animals, Inc., PA). The rats were deeply anesthetized with Nembutal $(50 \mathrm{mg} / \mathrm{kg}$, i.p.) and sequentially perfused through the ascending aorta with (1) $10 \mathrm{ml}$ of normal saline $(0.9 \%)$ containing 1000 units/ril heparin; (2) $50 \mathrm{ml}$ of $3.75 \%$ acrolein (Polysciences) and $2 \%$ paraformaldehyde in $0.1 \mathrm{M}$ phosphate buffer (pH 7.4); and (3) $200 \mathrm{ml}$ of $2 \%$ paraformaldehyde in $0.1 \mathrm{~m}$ phosphate buffer. The regions of the forebrain containing the hippocampal formation (as described by Paxinos and Watson, 1986) were removed and cut into coronal blocks 4 $\mathrm{mm}$ thick and stored in the latter fixative for an additional $30 \mathrm{~min}$. Sections ( $40 \mu \mathrm{m}$ thick) were then cut on a Vibratome and collected in $0.1 \mathrm{M}$ phosphate buffer and treated with $1 \%$ sodium borohydride prior to immunocytochemical labeling as described previously (Milner and Bacon, 1989).

Antisera. A polyclonal antiserum to GABA-hemocyanin conjugates supplied by Dr. Andrew C. Towle (Lab. of Molecular Neurobiology, Burke Rehabilation Center, 785 Mamaroneck, White Plains, NY 10605) was produced in rats by previously described methods (Lauder et al., 1986). Sprague-Dawley rats (Hilltop Lab Animals, Inc., PA) were immunized with GABA-glutaraldehyde-hemocyanin conjugates, prepared by the method of Storm-Mathisen et al. (1983). In tests for specificity, the immunoreactivity visualized in sections of tissue was not blocked by $10 \mathrm{~mm}$ unconjugated GABA or $100 \mu \mathrm{M}$ BSA-conjugated glutamate, $\beta$-alanine, or taurine but was completely abolished by preincubation with $10 \mu \mathrm{M}$ GABA-BSA (Lauder et al., 1986). The antiserum specificity was similar to that described previously by Hodgson et al. (1985).

A polyclonal antiserum to trypsin-treated $\mathrm{TH}$ was supplied by $\mathrm{Dr}$. Cory Abate (Department of Molecular Oncology, Roche Institute of Molecular Biology, Nutley, NJ). It was produced in rabbits against TH purified from the bovine adrenal medulla by methods described previously (Joh and Goldstein, 1973; Joh and Ross, 1983). The specificity of the antiserum for TH was demonstrated by Western blotting and by immunotitration. The antibody recognized a single band of protein in Western blots which in crude rat brain extract corresponded to the molecular weight of TH $(60,000 \mathrm{Da})$ and specifically inhibited the catalytic activity of TH in crude rat brain homogenates (Joh and Ross, 1983).

Dual labeling for TH and GABA. Dual labeling for TH by immunoautoradiography and GABA by the immunoperoxidase tcchnique is a modification of the procedure of Pickel et al. (1986) and incorporates the double-bridging modification (Ordronncau et al., 1981) of the peroxidase-antiperoxidase (PAP) method of Sternberger (1979). The sections were incubated sequentially in (a) a 1:2000 dilution of the TH antiserum for 18-24 hr; (b) three 10 min washes; (c) ${ }^{125}$ I-labeled donkey anti-rabbit immunoglobulin (IgG) (Amersham, Arlington Heights, IL) at a 1:100 dilution of a solution having a radioactive concentration of $100 \mu \mathrm{Ci} / \mathrm{ml}$ for $1 \mathrm{hr}$; (d) $10 \mathrm{~min}$ washes until negligible radioactivity was detected in the wash solution; (e) a 1:4000 dilution of GABA antiserum for 18-24 hr; (f) three 10 min washes; (g) a 1:50 dilution of goat anti-rat IgG (Sternberger-Meyer) for $30 \mathrm{~min}$; (h) three $10 \mathrm{~min}$ washes; (i) a 1:100 dilution of rat PAP complex for $30 \mathrm{~min}$; (j) three $10 \mathrm{~min}$ washes; and $(\mathrm{k})$ repeat of steps $\mathrm{g}-\mathrm{j}$. The peroxidase reaction product was visualized following incubation of the tissues with 3,3'-diaminobenzidine (DAB; Aldrich) and hydrogen peroxide. All incubations were carried out at room temperature with continuous agitation. The diluents and washes separating each of the incubations were prepared with 0.1 M Tris-saline (pH 7.6). The diluents also contained $0.1 \%$ BSA.

Controls. Nonspecific interactions between the 2 antisera and the specificity of the reactions were tested by (1) separately using the immunoperoxidase procedure for GABA and the immunoautoradiographic procedure for $\mathrm{TH}$; (2) substitution of $0.1 \mathrm{M}$ Tris-saline for the GABA antiserum; (3) substitution of $0.1 \mathrm{M}$ Tris-saline for the TH antiserum; and (4) omission of both the GABA and TH antisera.

Processing for light and electron microscopic autoradiography. For light microscopy, the labeled sections were mounted on acid-cleaned slides coated previously with $0.5 \%$ gelatin. The sections were processed for autoradiography according to the procedure of Cowan et al. (1972) as described in detail previously (Pickel et al., 1986). The autoradiographic exposure periods ranged from 4 to $25 \mathrm{~d}$. The final autoradiographic preparations were examined and photographed using differential interference contrast (DIC) optics on a Nikon Microphot microscope. Camera lucida drawings were made using a drawing tube attached to a Nikon Labophot microscope.

For electron microscopy, the labeled sections were fixed for $2 \mathrm{hr}$ in $2 \%$ osmium tetroxide in $0.1 \mathrm{M}$ phosphate buffer, washed in phosphate buffer, and dehydrated through a graded series of ethanols, $100 \%$ ethanol-propylene oxide (1:1), and propylene oxide. Sections were then placed in Epon 812 between 2 sheets of Aclar plastic (Masurovsky and Bunge, 1968). Regions that were found to contain the most overlap of GABAergic perikarya and processes and TH-labeled processes by light microscopy were selected for electron microscopy and were embedded with Epon 812 in Beem capsules. Electron microscopic autoradiography was performed by the procedure of Beaudet (1982) and Beaudet and Descarries (1986) as described by Pickel et al. (1986). Briefly, ultrathin sections $(50 \mathrm{~nm})$ were collected with a loop and deposited on slides coated previously with $2 \%$ parlodion in amyl acetate. The slides were counterstained with uranyl acetate and Reynold's lead citrate (Reynolds, 1963) and then coated with a silver-gray layer of carbon (Varian Vacuum Evaporator). The slides were dipped in Ilford $\mathrm{L}-4$ emulsion $\left(50^{\circ} \mathrm{C}\right) \mathrm{di}-$ luted 1:4 with water, air-dried, and exposed in light-proof boxes for periods of 6-15 months. The autoradiographs were developed 1.5-2 min with Kodak Microdol-X developer $\left(17^{\circ} \mathrm{C}\right)$, rinsed in water, and fixed for $4 \mathrm{~min}$ in $30 \%$ sodium thiosulfate. The thin sections on the parlodion coating were collected on grids that were subsequently immersed for $3 \mathrm{~min}$ in amyl acetate.

Final preparations of autoradiographic thin sections were examined with a Philips 301 electron microscope. Electron microscopic analysis was conducted on 10 plastic-embedded, double-labeled sections collected through rostrocaudal portions of the DG and CA3 of the hippocampus. The selection of these sections was based on optimal preservation of morphological details and maximal detection of both TH- and GABA-like immunoreactivity as observed by both light and electron microscopy.

Rationale for experimental design and controls. In the electron micrographs, immunoautoradiographic labeling for $\mathrm{TH}$ was visualized more easily than the peroxidase labeling for GABA. This was due in part to the overlying emulsion layer, which obscured the visualization of the peroxidase reaction product, and also to the greater sensitivity of the ${ }^{125} \mathrm{I}$-marker. Thus, to insure that the analysis would not be biased towards one of the labels, only those micrographs that contained profiles labeled with both markers were used in the final quantitative analysis.

The peroxidase reaction product within the GABA-labeled processes often appeared pale beneath the overlying emulsion and could be recognized largely by comparison with other unlabeled processes in the surrounding neuropil. Thus, the morphology and types of junctions 
formed by GABAergic neurons were examined first in sections not processed for autoradiography where the PAP reaction product was more easily visualized.

In the electron microscopic autoradiographs, the profile of the suspected source of radiation was identified by the presence of silver grains in at least 2 adjacent thin sections in order to differentiate specific autoradiographic labeling from background labeling. Additionally, a modification of the method of Salpeter et al. (1978) for quantitatively evaluating the distribution of silver grains was performed. For this, immunoautoradiographs were examined for the identity of cellular profiles that were the most probable sources of the observed silver grains indicative of the immunolabeling for TH. Initial photomicrographs from the hippocampal formation suggested that the primary structures that contained silver grains were axons and axon terminals. This was verified quantitatively in 60 randomly chosen micrographs in which 287 silver grains were individually assessed with regard to their cellular origin. Of these, $81 \%$ had central points located within the area bounded by the plasmalemmas of axons and axon terminals and $12 \%$ had central points located within a $150 \mathrm{~nm}$ radius of the terminal plasmalemmas. The remaining $7 \%$ of the silver grains had central points that were greater than $150 \mathrm{~nm}$ from the outer plasmalemmas of the labeled profiles. Since the terminals usually had less than 4 grains within single sections, the detection of silver grains over the same profile in a least 2 serial sections was considered as the minimum requirement for establishment of immunoautoradiographic labeling.

\section{Results}

\section{Light microscopy}

Within the hippocampal formation, GABA-LI, as indicated by the brown peroxidase reaction product, was found in neuronal perikarya and processes. In agreement with previous studies, immunocytochemically localizing GABA as well as GAD (Ribak et al., 1978; Sloviter and Nilaver, 1987), the regions containing the highest density of GABA-labeled perikarya and processes were in the infragranular hilus of the DG, in strata oriens, pyramidale, lucidum, and radiatum of $\mathrm{CA} 3$ of the hippocampus, and in strata pyramidal and radiatum of CA1 of the hippocampus. Numerous clumps of black silver grains indicative of processes immunolabeled for $\mathrm{TH}$ were observed interspersed between GABA-labeled perikarya and processes in the infragranular hilus of the DG (Fig. 1, $B-D$ ) and in strata pyramidale, lucida, and radiatum of $\mathrm{CA} 3$ of the hippocampus (Fig. 1, $E, F$ ). Occasionally, a TH-labeled process was found in close proximity to perikarya containing GABA-LI (Fig. 1, C,F). Although less numerous, processes with $\mathrm{TH}$ immunoreactivity were found in the same regions as GABA-labeled neurons and processes in the molecular layer of the DG (Fig. $1 A$ ) and in stratum oriens of CA3.

\section{Electron microscopy \\ Morphology of $G A B A$-ergic neurons in the $D G$ and $C A 3$ of the hippocampus}

In both the DG and CA3 of the hippocampus, GABA-LI was found in neuronal perikarya, dendrites, axons, and axon terminals (Figs. 2-8). Since the morphological characteristics of the GABA-labeled neurons did not differ significantly between the 2 regions, the results will be discussed together. The GABAcontaining perikarya were either ovoid or round, contained abundant cytoplasm, and had medium (15-25 $\mu \mathrm{m}$ ) to large (25$40 \mu \mathrm{m}$ ) diameters (Fig. 2A). The nuclei were unlabeled, had an indented nuclear envelope, and usually contained one nucleolus in single sections. Mitochondria, rough and smooth endoplasmic reticulum, and Golgi apparatus were the most distinguishablc cytoplasmic organelles.

GABA-LI also was seen in large (1.5-3.0 $\mu \mathrm{m}$ in diameter) proximal dendrites and in small $(0.5-1.5 \mu \mathrm{m}$ in diameter) distal dendrites. Proximal dendrites were similar to the perikarya in that they contained mitochondria, ribosomes, and rough endoplasmic reticulum as well as microtubules (Figs. $3 C, 5 A$ ). Smaller dendrites usually contained only mitochondria and microtubules (Figs. $3, A, B ; 4 D ; 6$ ).

The majority of axons with GABA-LI were small (0.1-0.2 $\mu \mathrm{m}$ in diameter) and unmyelinated (Fig. $4 D$ ). Occasionally, larger $(0.8-1.2 \mu \mathrm{m}$ in diameter) myelinated axons with GABA-LI were observed (Fig. 4C). These myelinated axons showed many laminae of myelin and were the same size as most of the unlabeled myelinated axons found in the same region.

The GABA-containing terminals ranged from $0.3-3.0 \mu \mathrm{m}$ in diameter and contained a few mitochondria and numerous small clear vesicles (scvs) (Figs. $2 C ; 3 C ; 4 ; 7-8$ ). Sometimcs, the tcrminals with GABA-LI also contained 1 or 2 large, dense-core vesicles (dcvs). Moreover, a few terminals with GABA-LI also contained TH-I (Fig. 7, $A, B$ ).

\section{Associations of GABA-labeled neurons in the $D G$}

Perikarya and dendrites with GABA- $L I$ were "associated" with terminals containing GABA-LI, TH-I, or lacking either label. (The term "associated" includes all synapses and appositions not separated by glia.) Out of 133 terminals included in the quantitative analysis, the majority $(62 \%)$ of the presynaptic terminals were unlabeled (Figs. $2, B, C, 3 ; 5, B, C$ ). The unlabeled terminals contained mitochondria, numerous scvs, and a few dcvs. These formed both symmetric and asymmetric junctions. The GABA-labeled perikarya and dendrites were usually postsynaptic to more than one unlabeled terminal in single section (Figs. $2 B ; 3, A, C$ ).

GABA-labeled perikarya and dendrites were equally contacted by terminals with GABA-LI (19\%) and terminals with $T H-I(19 \%)$. GABA-containing terminals appeared principally to form appositions without apparent synaptic specializations with GABA-labeled perikarya (Fig. 2C) and symmetric membrane specializations with dendrites (Fig. 3C); however, a few asymmetric junctions also were observed on dendrites (not shown). However, the density of the peroxidase product at postsynaptic sites precluded the distinction between the types of synapses in other cases. Usually, the GABA-labeled perikarya were contacted by a single GABA-containing terminal (Fig. 2C).

\footnotetext{
Figure 1. Light microscopic localization of the GABA and TH antisera in the hippocampal formation of the adult rat. Perikarya and processes with the immunoperoxidase reaction product for GABA-LI are brown, whereas processes showing immunoautoradiographic label for TH are black. $A$, Occasionally, TH-labeled processes (small arrows) are in the same region as perikarya and processes (arrowheads) with GABA-LI in the molecular layer $(M L)$ of the dentate gyrus. $B-D$, In the dentate gyrus, the distribution of TH-labeled processes (small arrows) overlaps that of $\mathrm{GABA}$-containing perikarya in the infragranular zone of the dentate gyrus. Although some overlap is observed in the most rostral sections $(B)$, the majority is found caudally $(C, D) . E$ and $F$, In CA3, GABAergic perikarya are most often codistributed with TH-labeled processes (small arrows) in the pyramidal cell layer $(P C L)$ and strata lucidum $(S L U)(E)$ and radiatum $(S R)(F)$. In both regions, the processes with TH-I are found sometimes in close apposition (small double arrows) to GABA-labeled perikarya. Scale bars, $25 \mu \mathrm{m}$.
} 
A

B
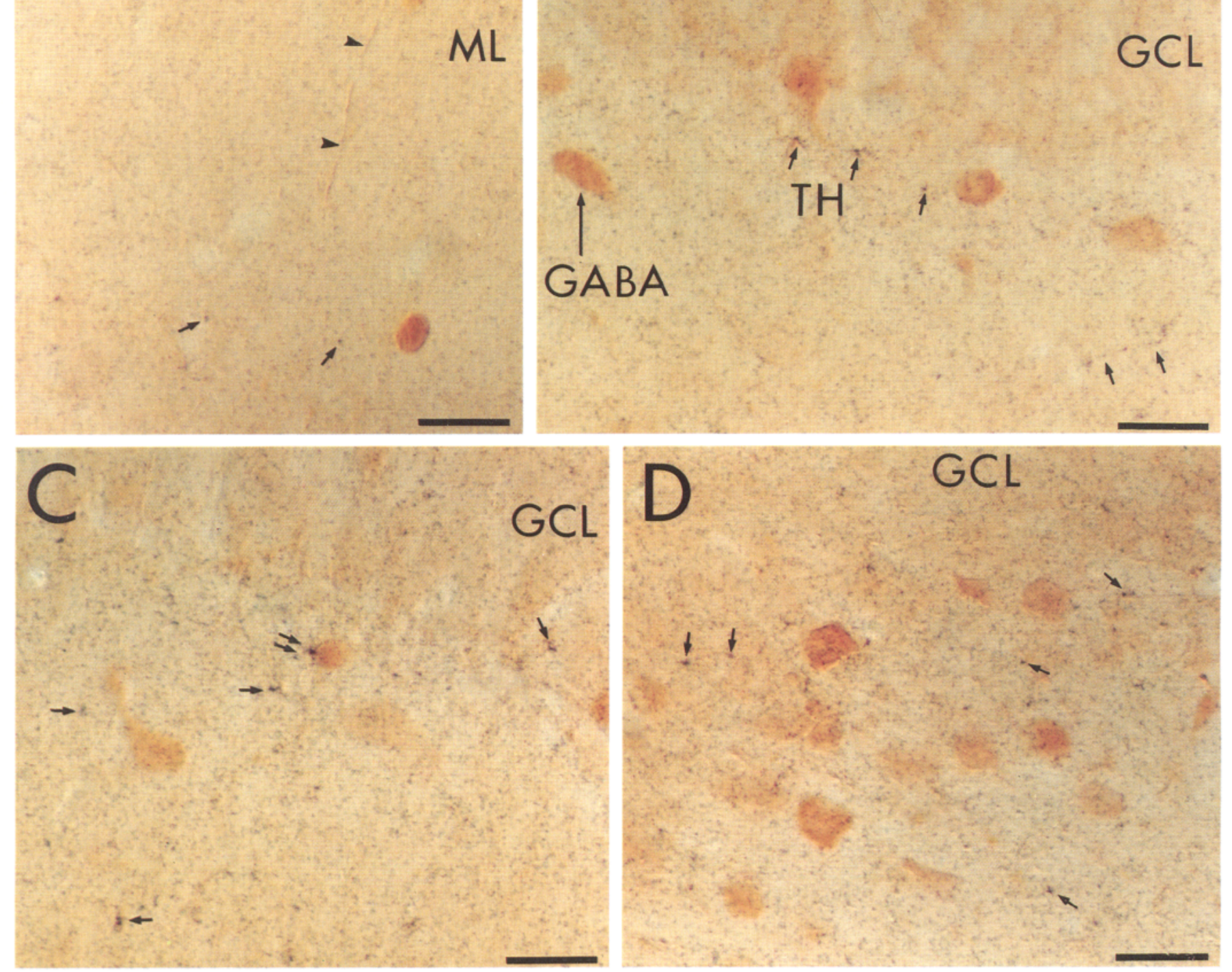

D

GCL

E

${ }_{\mathrm{PCL}} \mathrm{F}$

$S R$

i

SLU
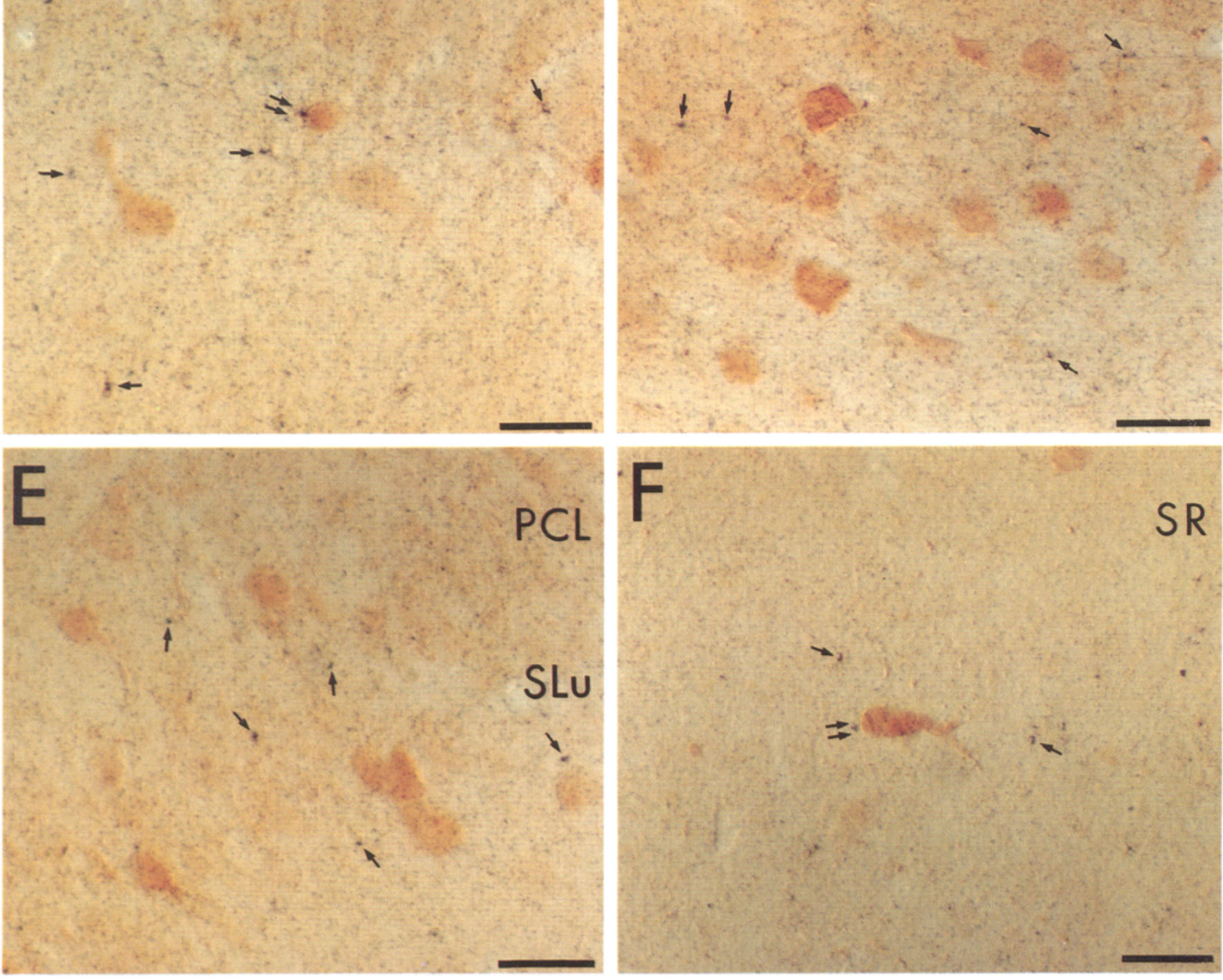
However, 3 or more GABA-labeled terminals also sometimes were associated with 1 labeled dendrite in single sections (Fig. $3 C$ ).

The terminals with TH-I rarely ( 2 out of 25 ) were associated with GABA-labeled perikarya. In these cases, the TH-labeled terminals either formed a symmetric junction or lacked an apparent membrane specialization in the plane of section analyzed. Whenever TH-labeled terminals were associated with GABAergic profiles, the majority were GABA-labeled dendrites (Fig. 5, A-C). Of the associations formed by these terminals, nearly half ( 10 out of 23 ) lacked any membrane specializations in the plane of section analyzed. However, in these cases the TH-labeled terminal and the GABA-containing dendrite were adjacent to each other without any apparent glial intervention (Fig. 5, $A-C$ ). The remainder of the TH-labeled terminals formed approximately equal proportions of symmetric and asymmetric junctions with unlabeled dendrites. Usually, only one TH-labeled terminal was associated with a GABA-labeled perikarya or dendrite; however, the same GABA-containing perikarya or dendrite was postsynaptic to other terminals that contained GABA-LI or lacked immunoreactivity for either TH or GABA (Fig. 5, $B, C$ ).

In quantitative evaluation of the neural associations of GABAlabclcd tcrminals, the majority were associated with perikarya and dendrites lacking immunoreactivity for either GABA or TH (see Table 1). Some of these unlabeled perikarya were identified as granule cells, based upon their localization in the granule cell layer. The somatic associations $(n=6)$ formed by the GABAcontaining terminals were characterized either as symmetric membrane specializations (Fig. $4 A$ ) or as lacking any junction in the plane of section analyzed.

More commonly ( 53 out of 59), the GABA-labeled terminals were associated with unlabeled dendrites (Figs. $4, B, D$ ). Some of the unlabeled dendrites originated from granule cell bodies; however, the cellular origin could not be ascertained in the majority of cases. The terminals were most often associated with the shaft portions of both large and small GABA-labeled dendrites. Of the total associations between GABA-containing ter- minals and unlabeled dendrites, $44 \%$ were of the symmetric type and the remaining $56 \%$ lacked a recognizable membrane specialization in the plane of section analyzed. Contacts on the unlabeled perikarya and dendrites from more than one GABAlabeled terminal were sometimes seen in single sections (Fig. 4, $A, D$ ). Moreover, convergence on the unlabeled perikarya and dendrites from terminals containing GABA-LI and terminals containing TH-I was seen in the same planc of section (see Table 1).

A significant number of the terminals exhibiting GABA-LI were associated with either soma or dendrites which also contained GABA-LI (Table 1). The rarely observed somatic associations were characterized by symmetric membrane specializations (Fig. 2C). The majority $(n=21)$ of the synapses formed by terminals with GABA-LI were on the shafts of both large and small GABA-labeled dendrites (Fig. 3C). Contacts formed between GABA-containing terminals and dendrites were equally divided between those that formed symmetric junctions and those that lacked a recognizable membrane specialization in the plane of section analyzed.

In the remaining $43 \%$ ( 65 out of 151 ) of the terminals with GABA-LI, some were not associated with any neuronal processes in the plane of section analyzed (see Table 1). These usually were separated from the neuropil by glial processes. Howcver, direct appositions between 2 axon terminals were often observed. These included appositions with other unlabeled terminals as well as those containing GABA-LI or TH-I (Table 1). In these cases, the terminals were in direct apposition to each other without any glial intervention.

\section{Associations of GABA-ergic neurons in CA3 of the hippocampus}

Compared with the DG, few perikarya with GABA-LI were found in CA 3 of the hippocampus. The majority of presynaptic terminals on the GABA-labeled perikarya and dendrites were nearly equally divided between terminals with $T H-I(39 \%$, or 10 out of 26) and terminals that lacked both TH-I and GABA$L I(46 \%$, or 12 out of 26$)$. Most of the terminals with TH-I were

Figure 2. Associations of unlabeled and GABA-containing terminals with GABA-labeled perikarya in the hippocampal formation. $A$, Low-
magnification electron micrograph depicts a perikaryon with GABA-LI in the CA3 region of the hippocampus. The perikaryon is small (10-15
$\mu \mathrm{m})$ and contains an indented nucleus $(N)$ and abundant cytoplasm. $E R$, endoplasmic reticulum; $G$, Golgi apparatus; $m$, mitochondria. $B$, Higher-
magnification electron micrograph shows a GABA-immunoreactive perikaryon $(G A B A-P)$ in the dentate gyrus postsynaptic to 2 unlabeled terminals
$(u T)$ which form asymmetric junctions $(c u r v e d$ arrows) or close associations without any glial interface $($ double straight arrows). $C$, Both an unlabeled
terminal $(u T)$ and a $G A B A$-containing terminal $(G A B A-T e)$ form synapses (closed and open arrows, respectively) with a perikaryon with $G A B A-$
LI in the hilus of the dentate gyrus. Sections were not processed for autoradiography. Scale bars: $A, 1 \mu \mathrm{m} ; B$ and $C, 0.5 \mu \mathrm{m}$.

Figure 3. Associations of unlabeled and GABA-labeled terminals with dendrites with GABA-LI in the dentate gyrus. $A$, A small GABA-labeled dendrite is shown which receives multiple symmetric contacts (arrows) from numerous unlabeled terminals $(u T)$. $B$, An unlabeled terminal $(u T)$ forms an asymmetric synapse (arrow) with a small dendrite with GABA-LI which also is associated (small arrows) with a small axon. $C$, Both GABA-containing and unlabeled terminals $(u T)$ form synaptic junctions (open and closed arrows, respectively) with a large GABA-containing dendrite. Sections were not processed for autoradiography. Scale bars, $0.5 \mu \mathrm{m}$.

Figure 4. Axosomatic and axodendritic associations between GABA-labeled terminals and unlabeled dendrites in the hippocampal formation. $A$, Two terminals with GABA-LI form symmetric synapses (open arrows) with an unlabeled perikarya $(u P$ ) in the hilus of the dentate gyrus. The GABA-labeled terminals contain numerous small, clear vesicles (scv). B, A GABA-containing terminal forms a symmetric synapse (open arrow) with a large unlabeled dendrite $(u D)$ in the hilus of the dentate gyrus. $C$, In CA3, a terminal with GABA-LI (TI) forms a symmetric synapse (open arrow) with an unlabeled dendrite which also receives an asymmetric contact (closed arrow) from an unlabeled terminal (uT). The same GABAlabeled terminal also is adjacent (arrowheads) to another GABA-labeled terminal (T2) without any glial intervention and to a myelinated axon $(m A)$ with GABA-LI. $D$, In the hilus of the dentate gyrus, a GABA-labeled terminal $(T I)$ forms a synapse (open arrow) with an unlabeled dendrite $(u D)$ and is also closely apposed (small arrows) to a GABA-containing dendrite (GABA-D). The unlabeled dendrite is also postsynaptic to both an unlabeled terminal $(u T)$ which forms an asymmetric synapse (closed arrow) and is closely apposed (small double arrows) to another GABA-labeled terminal (T2). Sections were not processed for autoradiography. Scale bars, $0.5 \mu \mathrm{m}$. 


\section{A \\ Alooes. $=0$}

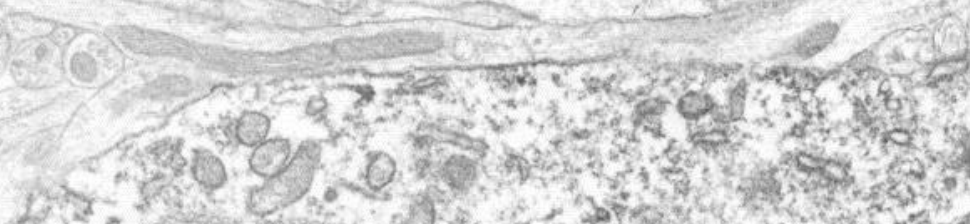

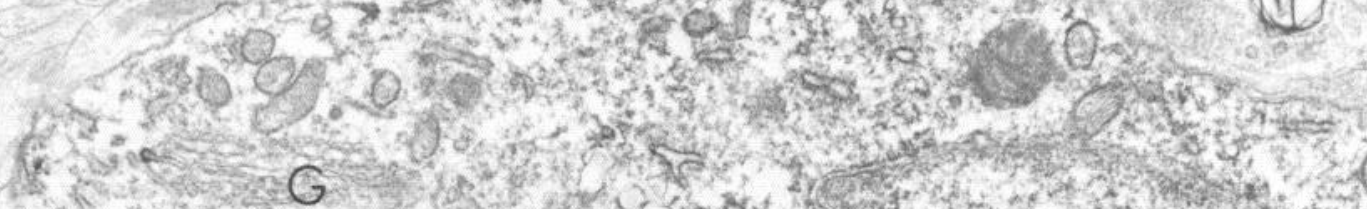

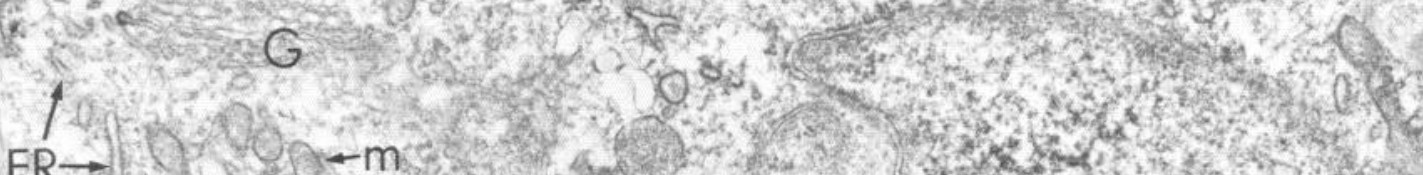

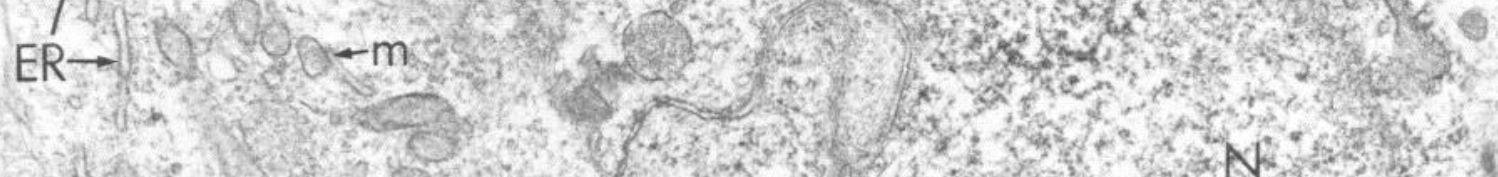

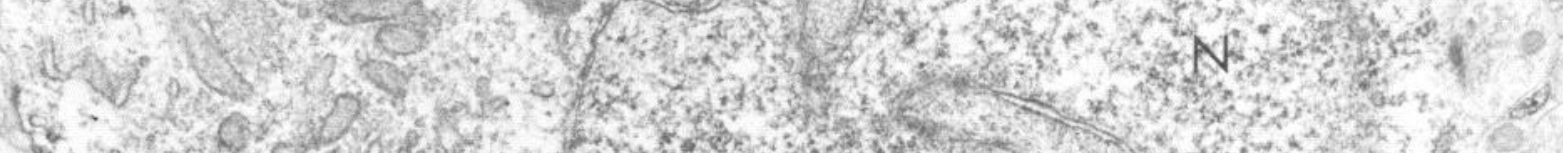
(5.

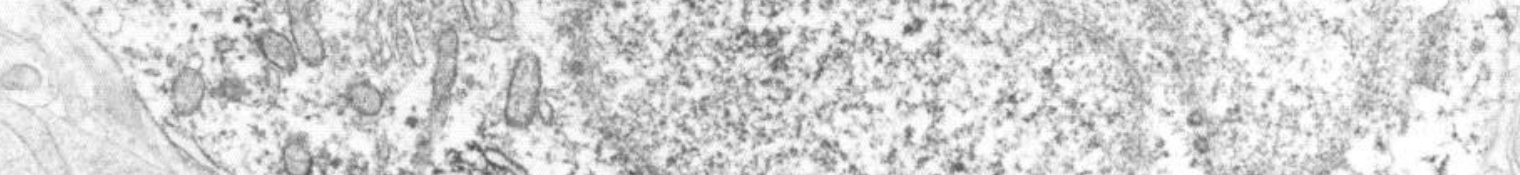

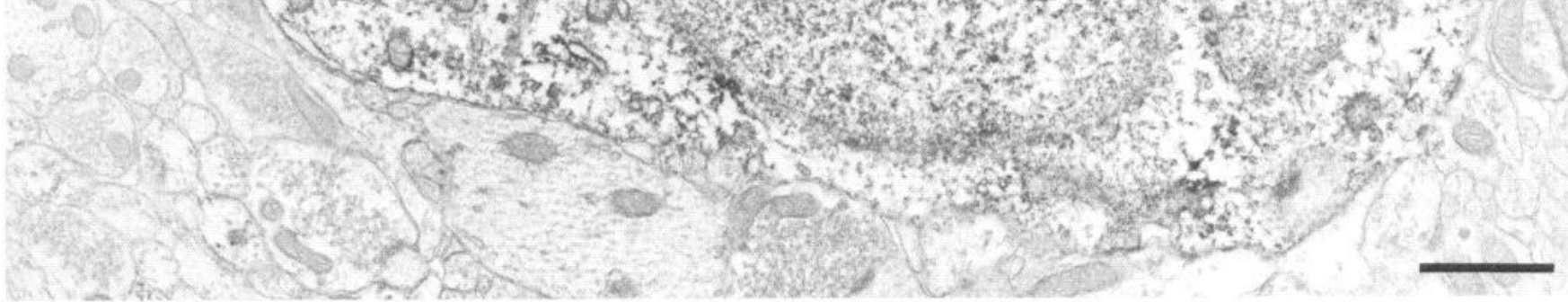

B

$$
\text { (1) }
$$

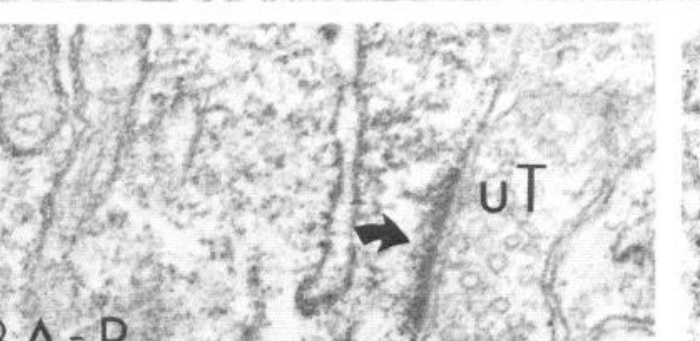

GABA P 1.1

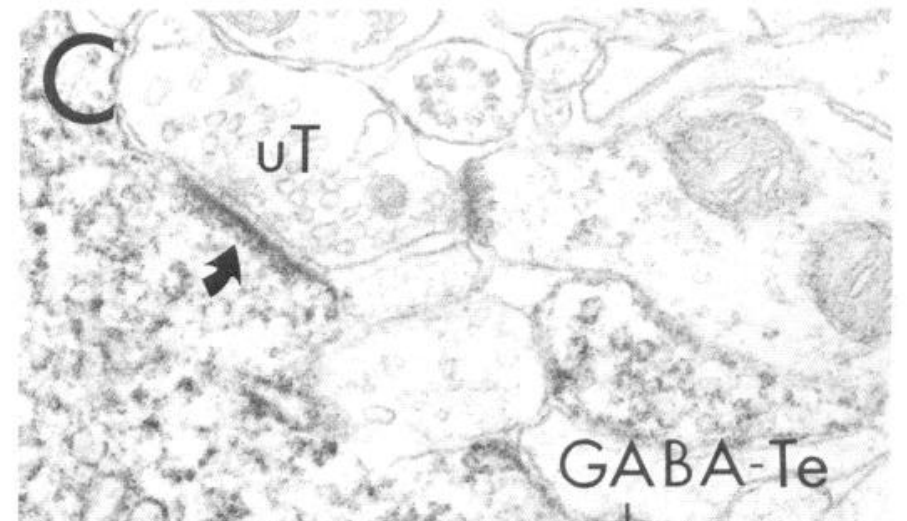

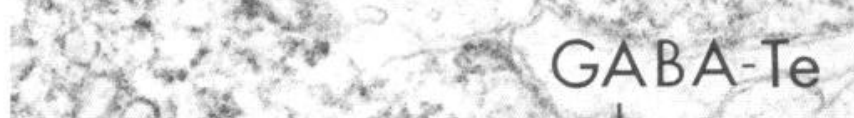

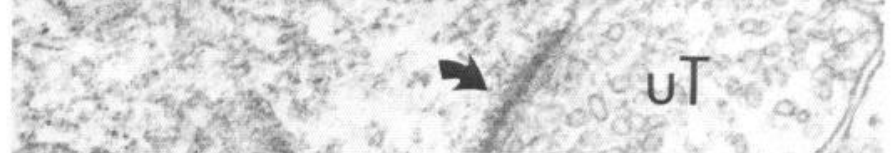
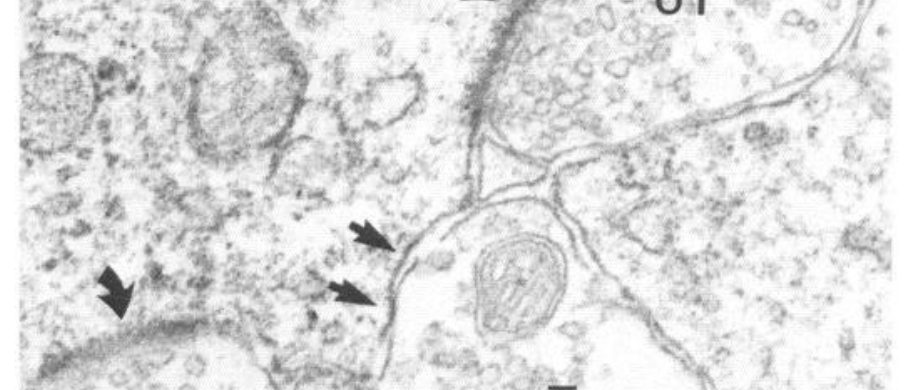

2 5.

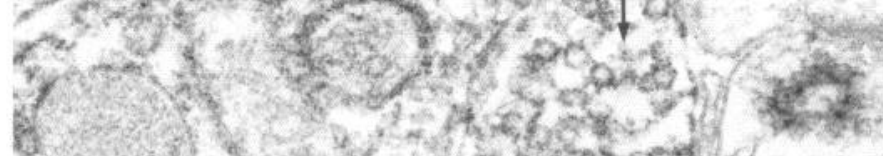
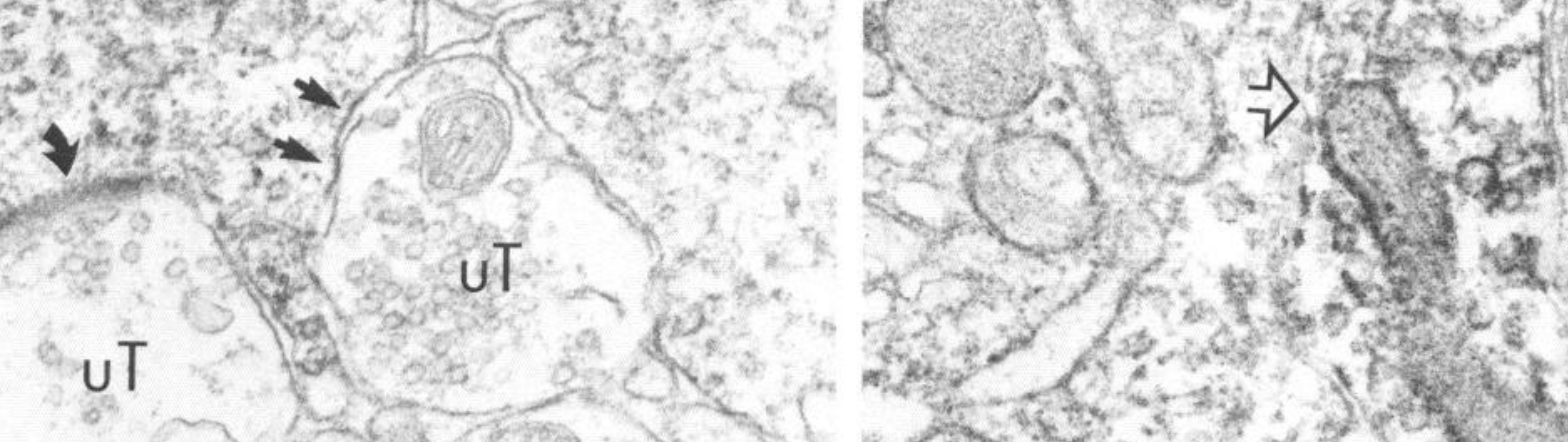

be

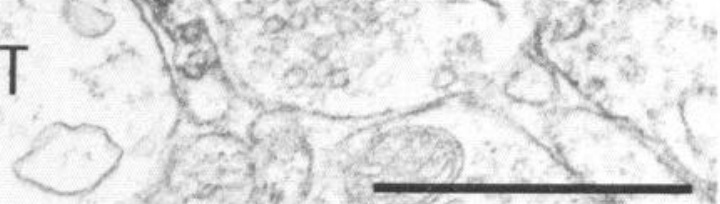

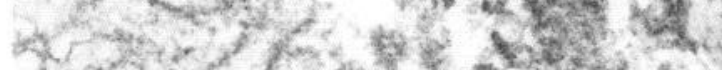

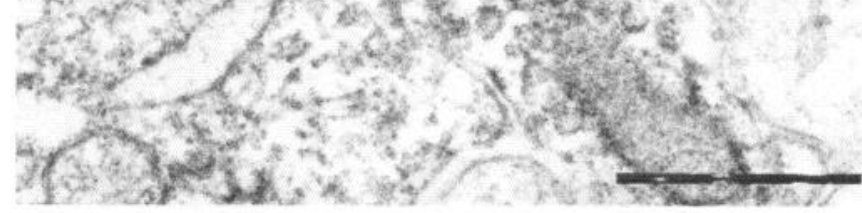



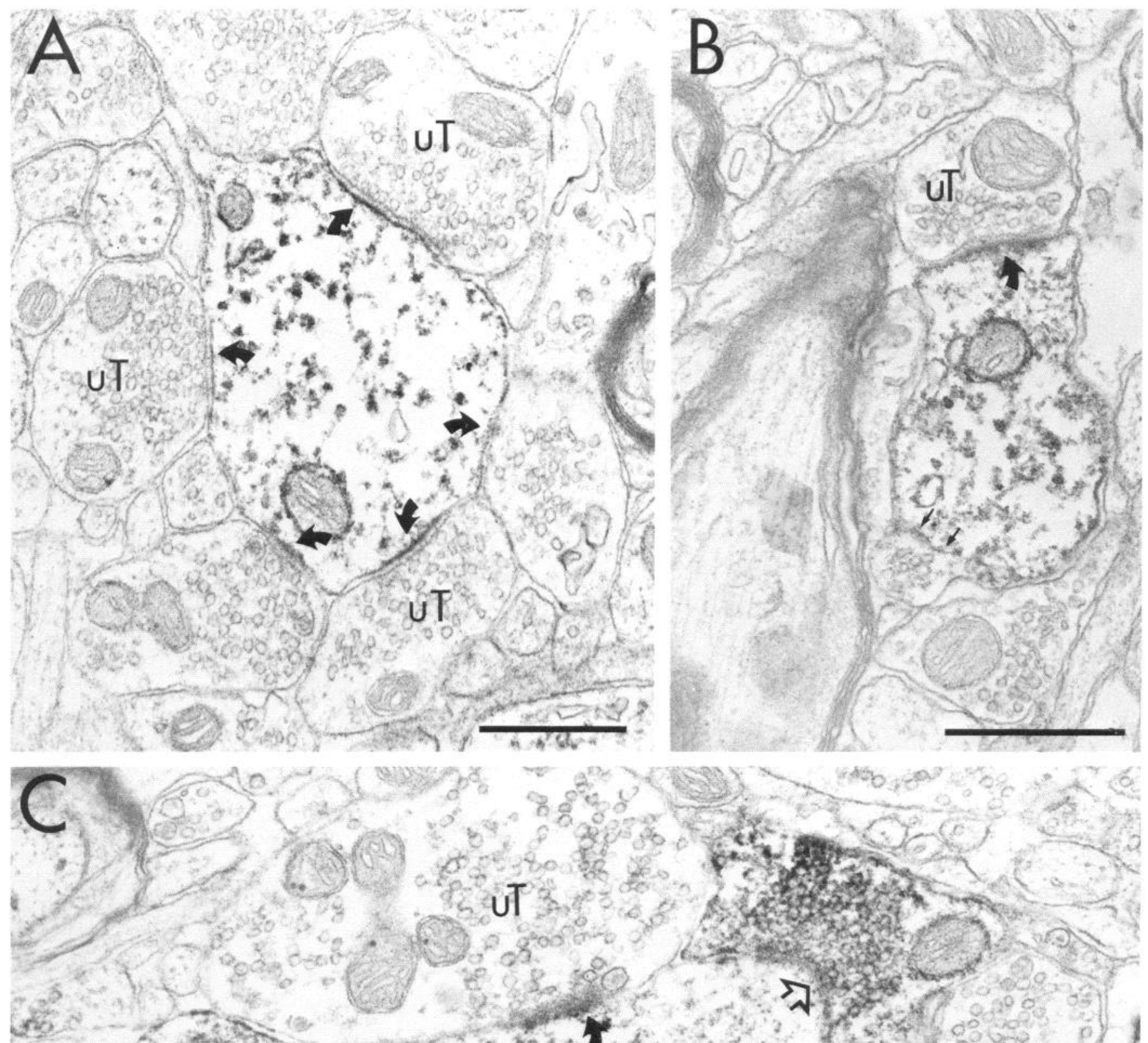

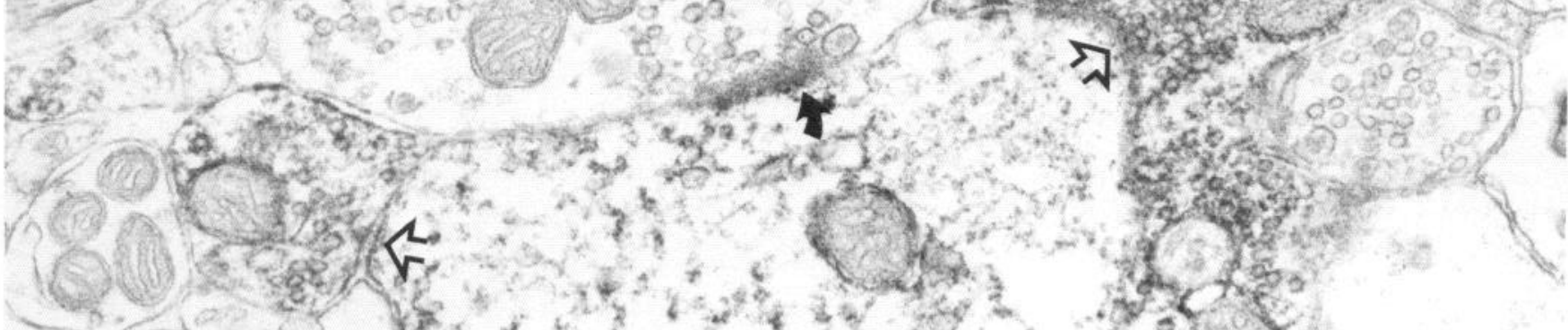

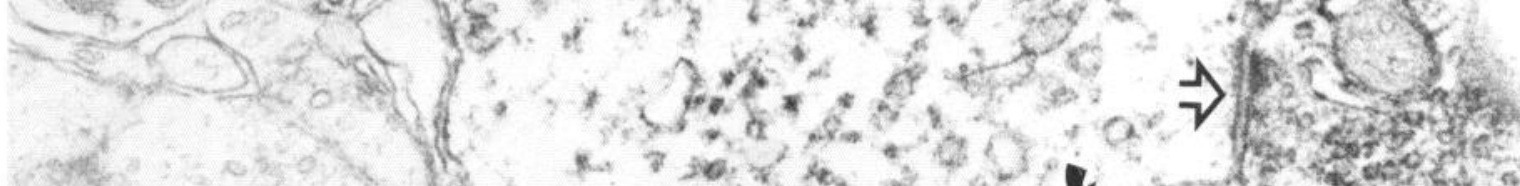

The 
A

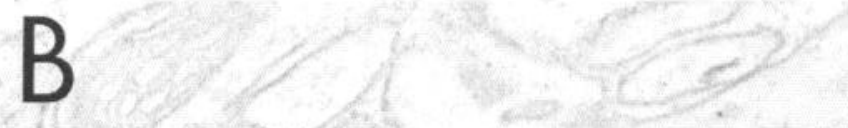

*

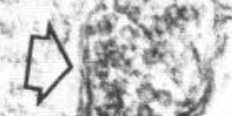

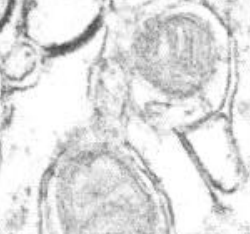

it.

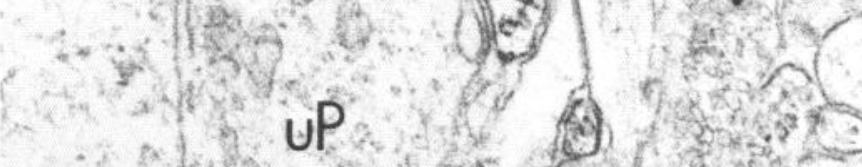

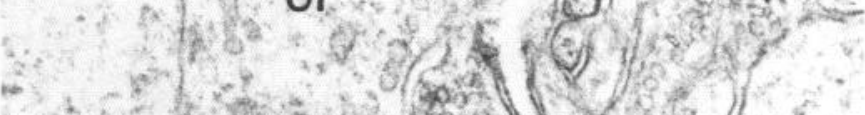

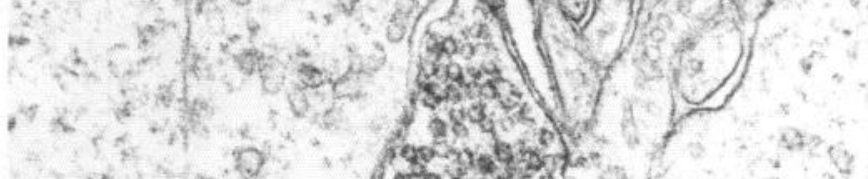

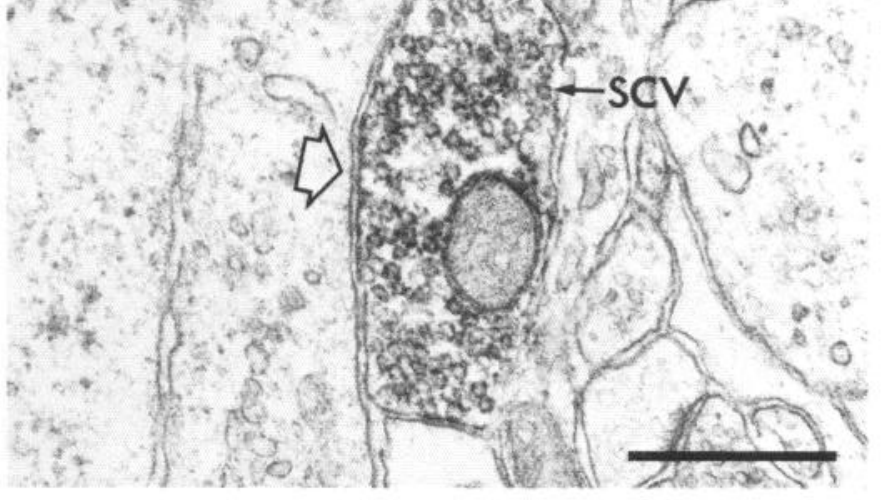

UT

- 8 की

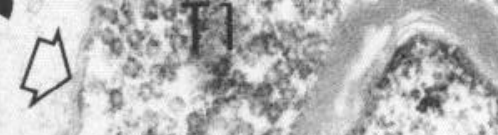

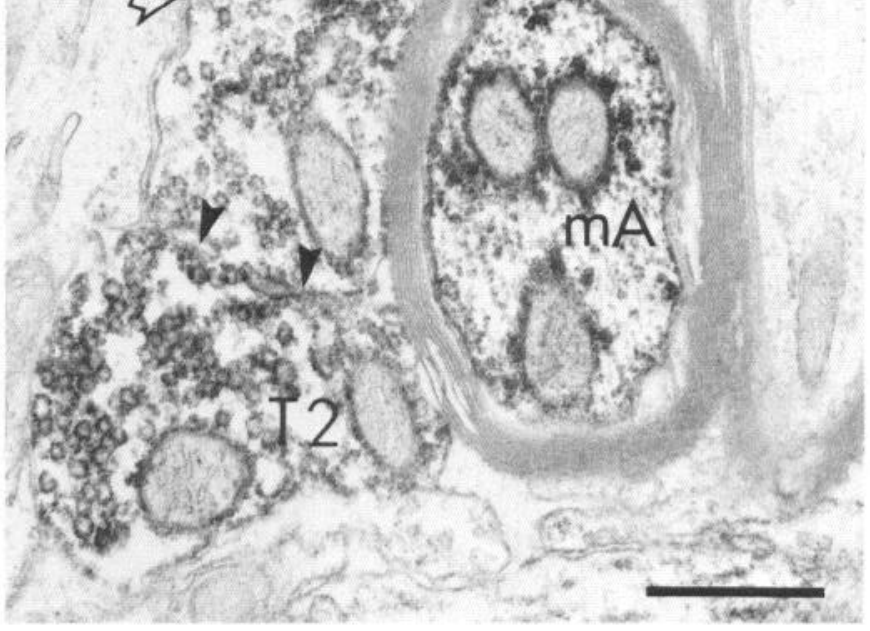

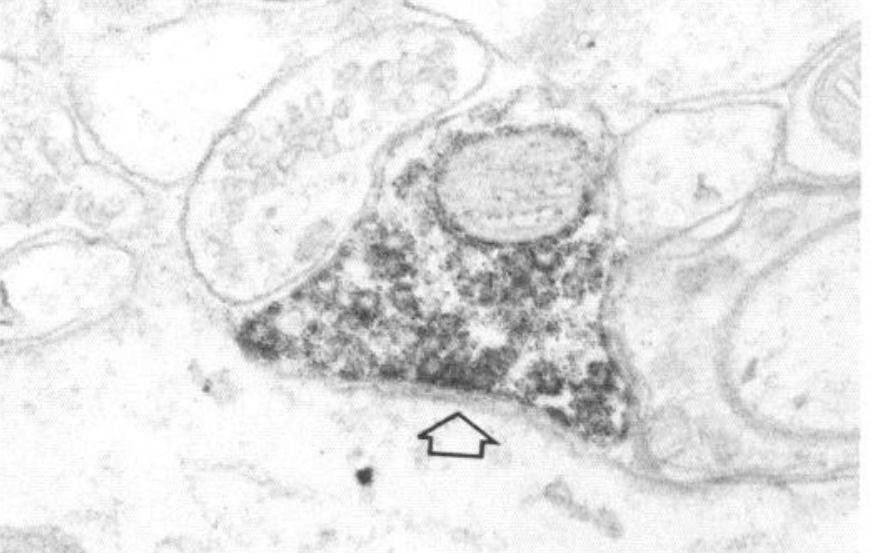

UD
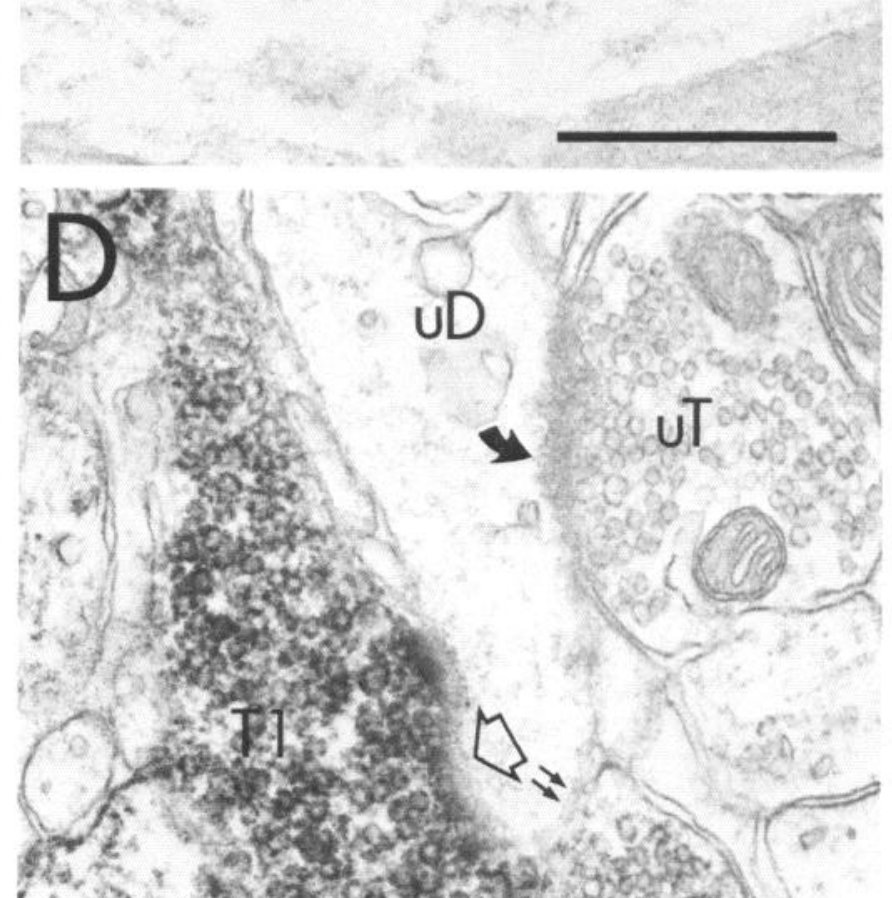

$\mathrm{GABA}$.

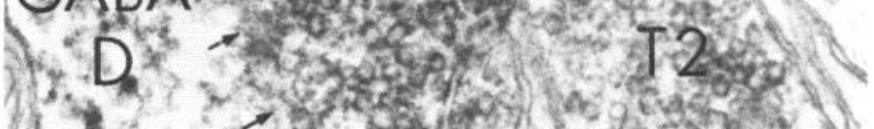

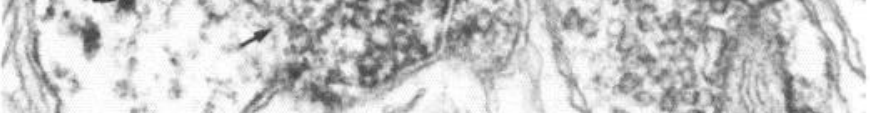

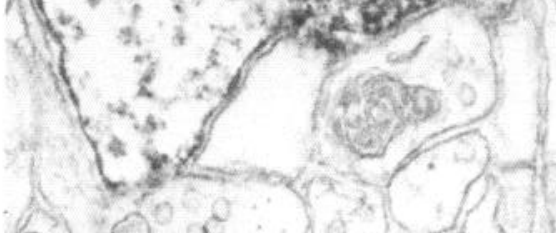

hi $00 \%$

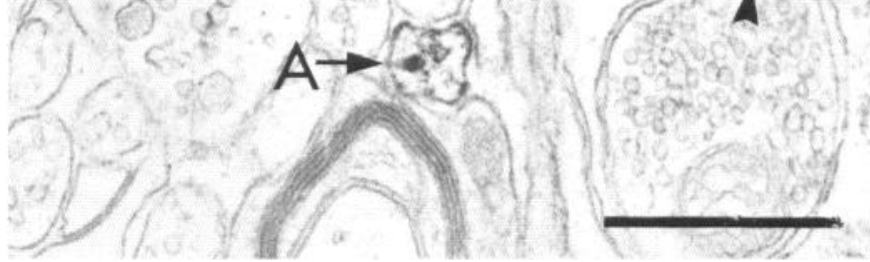

(cog)

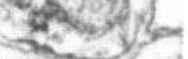



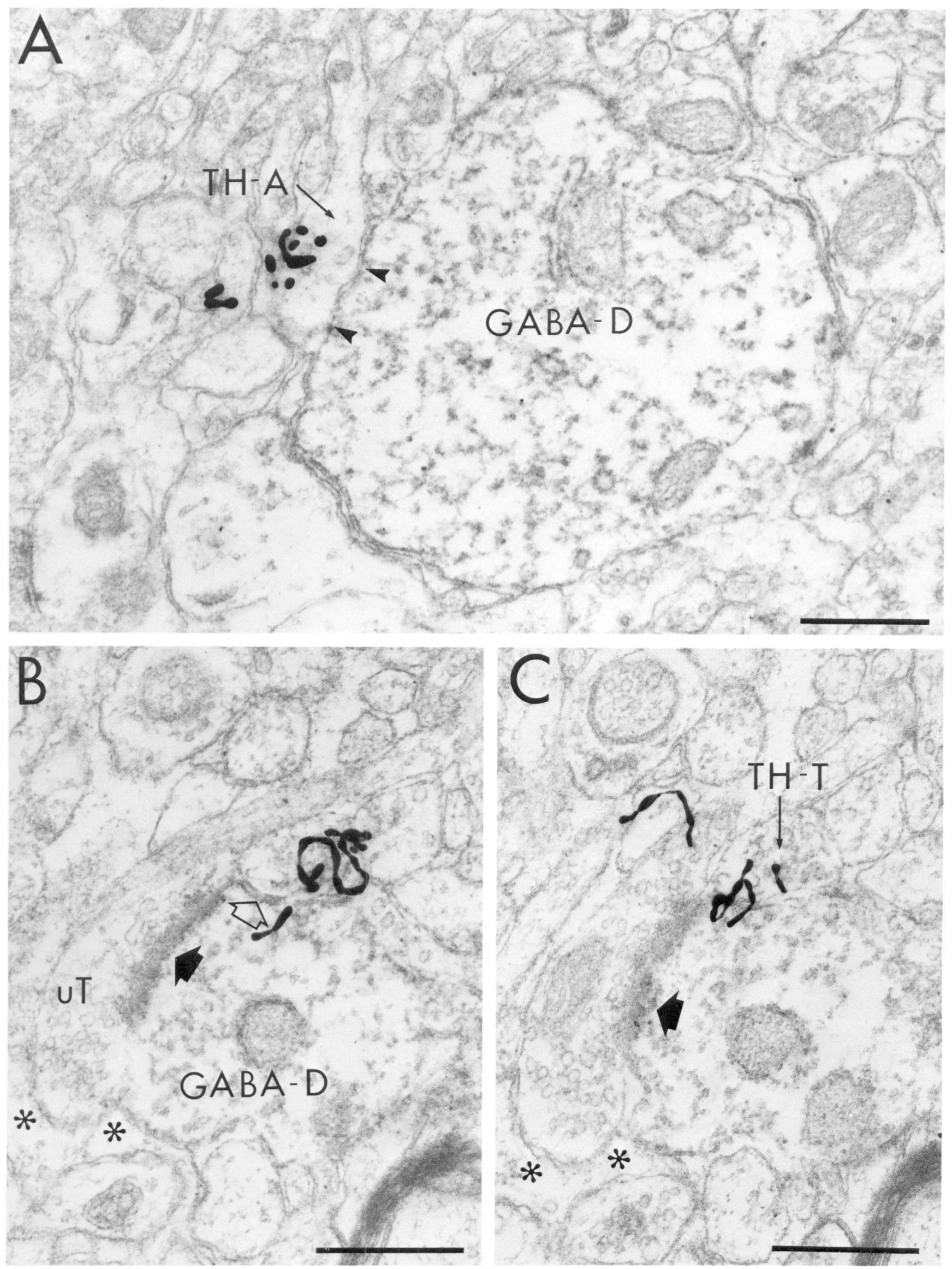


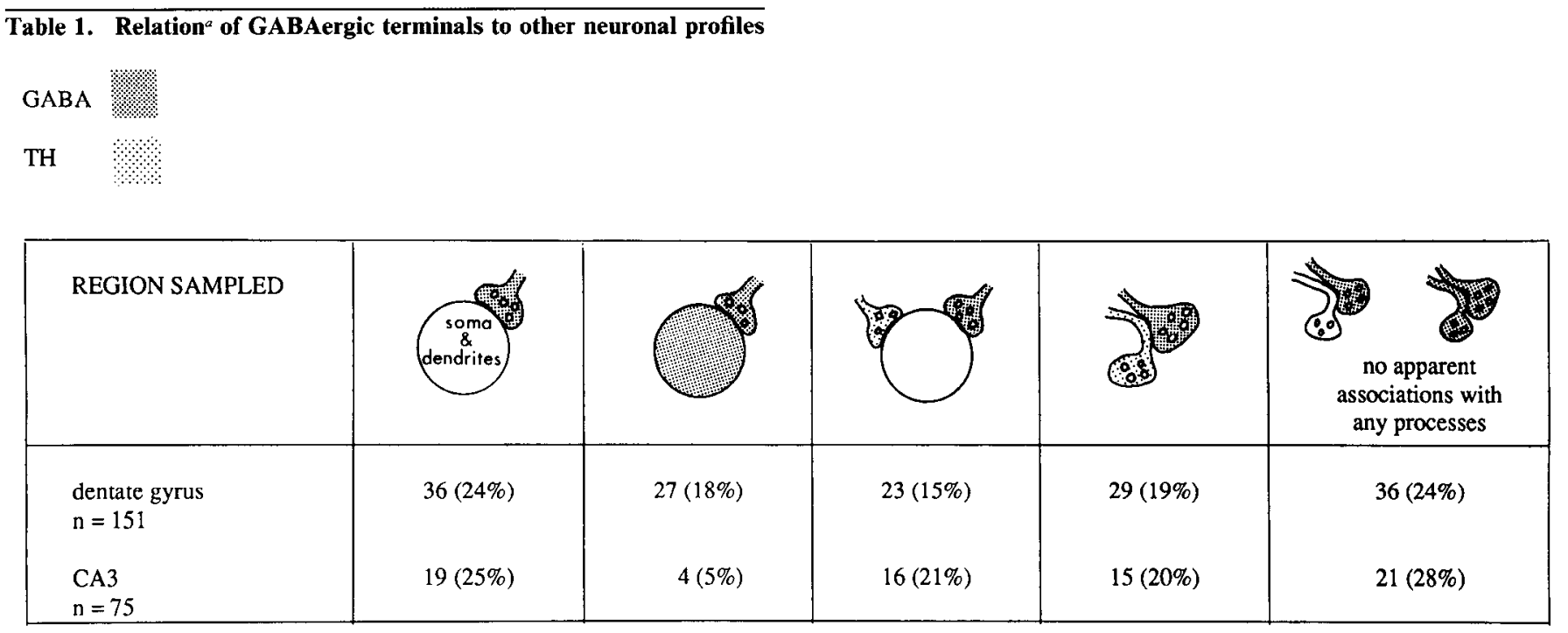

a Includes all synapses and appositions not separated by glia.

apposed to their targets without apparent glial intervention in the plane of section analyzed but did not form synaptic membrane specializations. However, asymmetric and symmetric junctions betwecn the TH-labcled terminals and GABA-labeled dendrites were sometimes found (Fig. 6). The GABA-labeled perikarya and dendrites were usually postsynaptic to only one $\mathrm{TH}$-containing terminal but more than one unlabeled terminal (as was pointed out in Fig. 5 from the DG). The unlabeled terminals contained mitochondria, numerous scvs, and a few devs and formed both symmetric and asymmetric junctions. The remaining $15 \%$ (4 out of 26 ) of the associations on GABAergic perikarya and dendrites were from terminals with $G A B A$ $L I$. In these cases, the terminals formed either symmetric junctions or lacked a recognizable membrane specialization in the plane of section analyzed. The GABA-containing perikarya and dendrites were often found postsynaptic to more than one GABAlabeled terminal.

In a quantitative analysis of the neuronal associations of GABA-labeled terminals, the majority formed synaptic contacts or appositions with unlabeled perikarya and dendrites (Table 1). The somatic synapses $(n=2)$ formed between GABA-containing terminals and unlabeled perikarya were characterized by symmetric membrane specializations. More commonly (33 out of 35), the terminals containing GABA-LI were associated with unlabeled dendrites (Fig. $4 \mathrm{C}$ ). Of these, the majority were on the shaft portion of large and small dendrites. Two-thirds of the GABA-labeled terminals were associated without intervening glial processes but with no synaptic membrane specialization in the plane of section analyzed. The remaining third of the contacts were primarily symmetric; however, an occasional asymmetric junction was observed. Multiple contacts from
GABA-labeled terminals on the unlabeled perikarya and dendrites were common. Moreover, convergence on the unlabeled perikarya and dendrites from terminals containing GABA-LI and tcrminals containing TH-I was often obscrved (Table 1; Figs. $7, A, B ; 8, A-C$ ).

Relatively few of the terminals exhibiting GABA-LI formed associations with soma or dendrites that also contained GABA$L I$ (Table 1). The associations were either characterized by a symmetric junction or lacked a membrane specialization in the plane of section analyzed. The classification of the type of synaptic junction was often difficult due to the density of the peroxidase reaction product in the postsynaptic target.

In the remainder of the terminals with GABA-LI, the majority lacked any apparent synaptic density within the plane of section analyzed (see Table 1). However, direct appositions between 2 axon terminals, inclusive of those with TH-I (Fig. 8, $A, B$ ) and GABA-LI (Fig. $4 C$ ) or lacking either immunoreactivity, were common. In these cases, the terminals were in direct apposition to each other without any glial intervention.

\section{Discussion}

The results of this study provide information on the synaptic relations between GABAergic neurons and catecholaminergic terminals as well as other unlabeled neurons in the hippocampal formation. Specifically, we have shown that: (1) the distribution of GABAergic neurons overlaps that of catecholaminergic terminals primarily in CA3 of the hippocampus and the DG; (2) GABA-containing neurons receive inputs from unlabeled as well as from GABA- and TH-containing terminals; (3) GABAergic and catecholaminergic terminals synapse on common targets;

\footnotetext{
Figure 5. Associations between TH-labeled axons and terminals and GABA-labeled dendrites in the hilus of the dentate gyrus. $A$, A TH-labeled axon $(T H-A)$ is in close apposition (arrowheads) without any glial intervention with a large GABA-containing dendrite (GABA-D). The autoradiographic label in the TH-labeled terminal was confirmed in an adjacent section (not shown). $B$ and $C$, Serial electron micrographs showing a TH-labeled terminal ( $T H-T$ ) which forms a symmetric synapse (open arrow) with a small GABA-labeled dendrite (GABA-D). The GABA-containing dendrite is also postsynaptic to an unlabeled terminal $(u T)$ which forms an asymmetric synapse (closed arrow). This latter synaptic association is partially surrounded by a glial process (asterisk). Autoradiographic exposure, 15 months. Scale bars, $0.5 \mu \mathrm{m}$.
} 


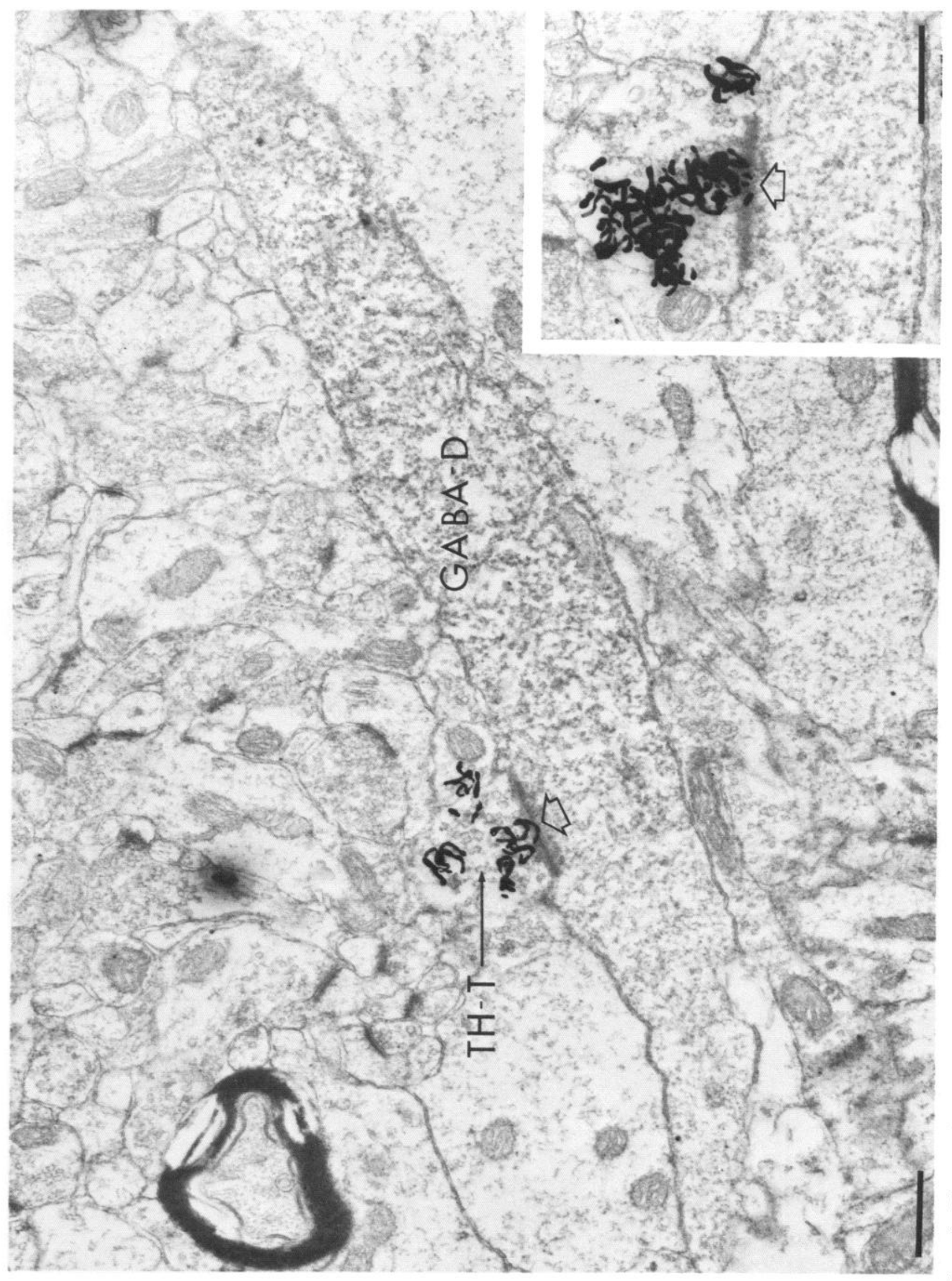



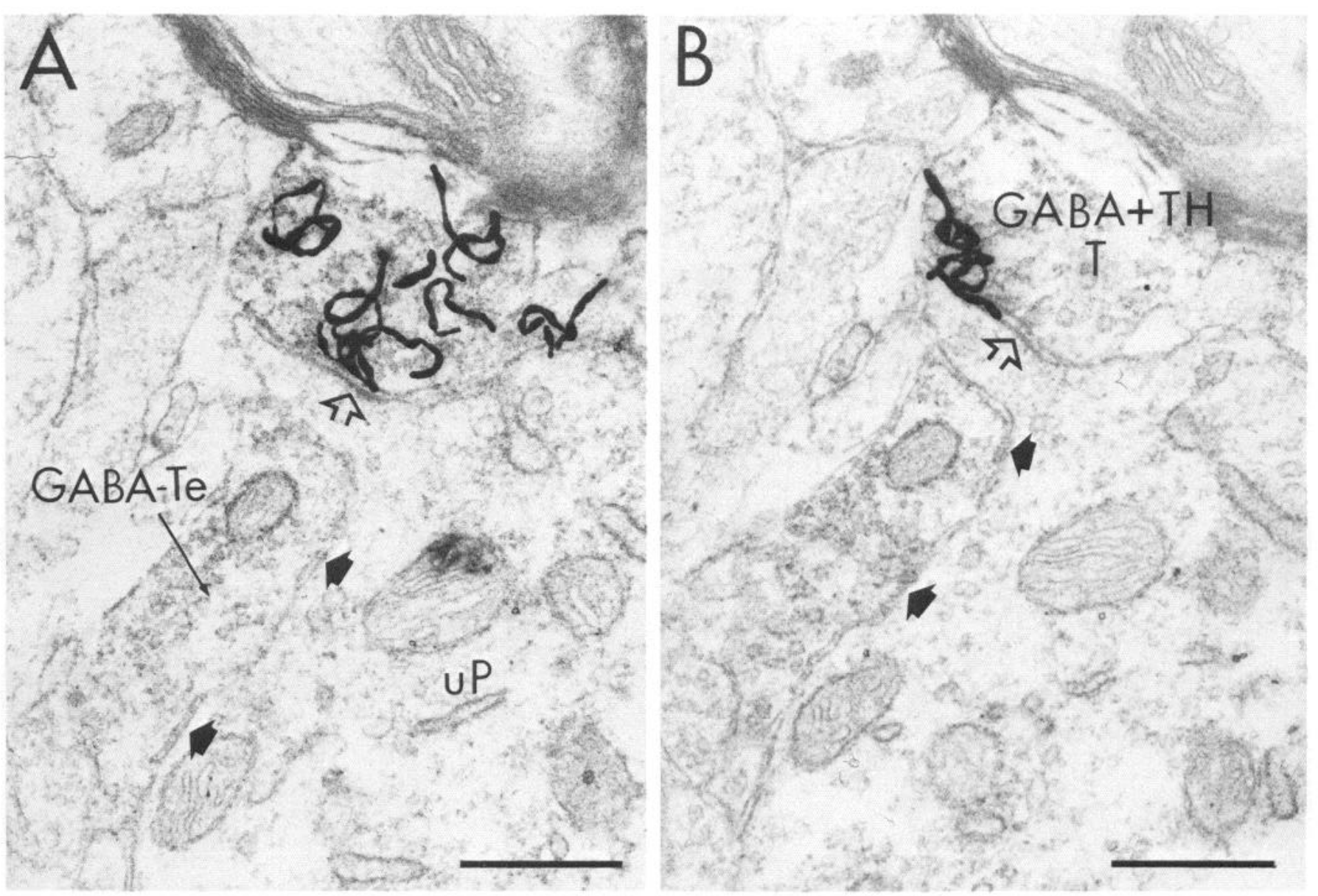

Figure 7. $A$ and $B$, Serial electron micrographs from stratum radiatum of CA3 show a terminal with GABA-LI $(G A B A-T e)$ which forms symmetric contacts (closed arrows) with an unlabeled perikarya $(u P)$ which also receives a symmetric contact (open arrow) from a terminal which contains both GABA-LI and TH-I $(G A B A+T H-T)$. Autoradiographic exposure, 15 months. Scale bars, $0.5 \mu \mathrm{m}$.

and (4) GABAergic and catecholaminergic terminals occur in apposition to each other.

\section{Methodological considerations}

\section{Dual-labeling technique}

We have used a technique for visualizing 2 antigens in single sections by combining peroxidase and immunoautoradiographic labeling of 2 antisera raised in separate species. This approach has been used previously for the combined localization of substance $\mathrm{P}$ or leucines-enkephalin and catecholamine-synthesizing enzymes in the rostral ventrolateral medulla (Milner et al., 1988, 1989) and is a modification of the method described previously by Pickel et al. (1986) for the dual localization of antisera from the same species. The advantages of the use of antisera from 2 different species have been addressed previously (Milner et al., 1988, 1989) and thus will not be discussed here.

\section{TH as an indicator of catecholamines}

The immunoautoradiographic localization of $\mathrm{TH}$ in axons and axon terminals that are primarily in the infragranular zone of the DG and strata lacunosum-moleculare and radiatum of the CA3 region of the hippocampus is consistent with our earlier studies utilizing peroxidase methods (Milner and Bacon, 1989). Additionally, the morphological characteristics of the TH-containing terminals as well as the types and distribution of synaptic contacts on the target neurons were similar to those identified previously by peroxidase labeling methods (Milner and Bacon, 1989). The relative distribution of catecholaminergic axons and terminals using TH immunocytochemistry is similar to that described using catecholamine histofluorescence (Loy et al., 1980) or dopamine- $\beta$-hydroxylase immunocytochemistry (Swanson and Hartman, 1975), suggesting that the majority of TH-labeled profiles are noradrenergic. However, as the enzyme catalyzing the first step in catecholamine synthesis, TH is found in dopaminergic as well as noradrenergic and adrenergic processes (Joh and Ross, 1983; Pickel and Milner, 1987). Thus, although most of the catecholaminergic innervation in the hippocampal formation is noradrenergic (for review, see Fallon and Loughlin, 1987), the fact that TH immunoreactivity reflects the sparse dopaminergic innervation (Pohle et al, 1984; Verney et al., 1985) cannot be disregarded.

\section{Relative distribution and morphology of GABA neurons}

By light microscopy, perikarya and processes immunoreactive for GABA were primarily in the hilus of the DG and strata radiatum and lacunosum-moleculare of the hippocampus. This distribution is consistent with previous studies in the same or different species which similarly used antibodies to GABA (Gamrani et al., 1986; Peterson and Ribak, 1987; Sloviter and Nilaver, 1987; Babb et al., 1988) or to glutamic acid decarbox- 
$A$ त A 7 GABA B 2 . $G A B A T S \quad 4=2$

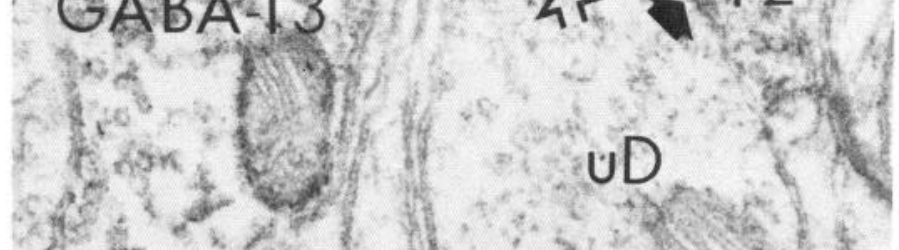
$6 y+2+\frac{10}{2}$
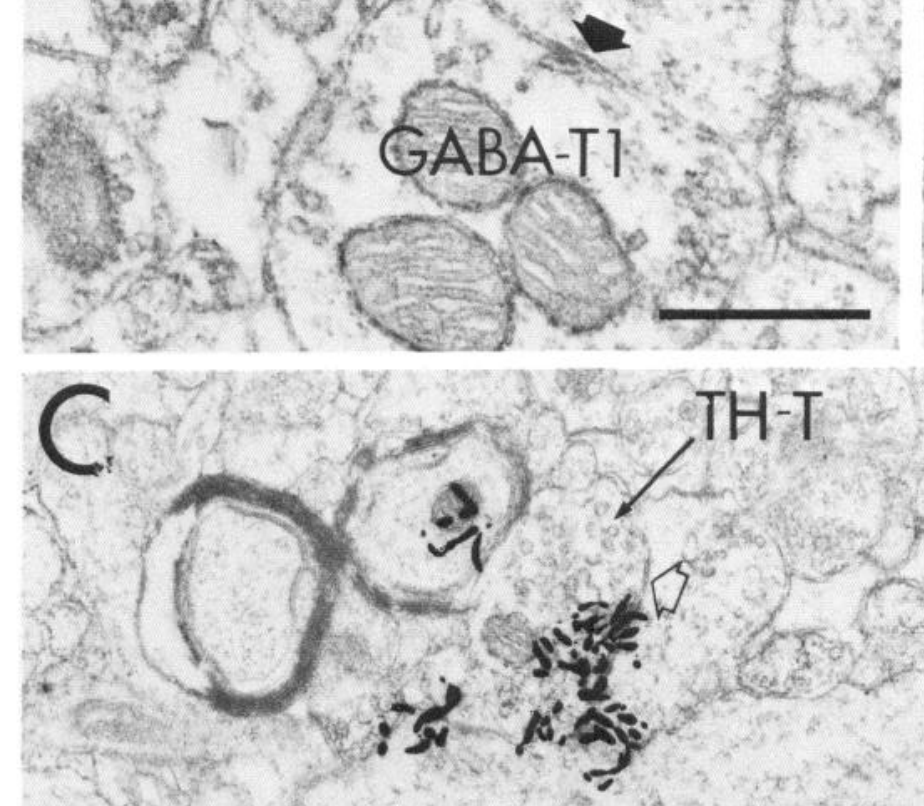

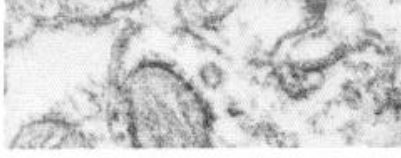

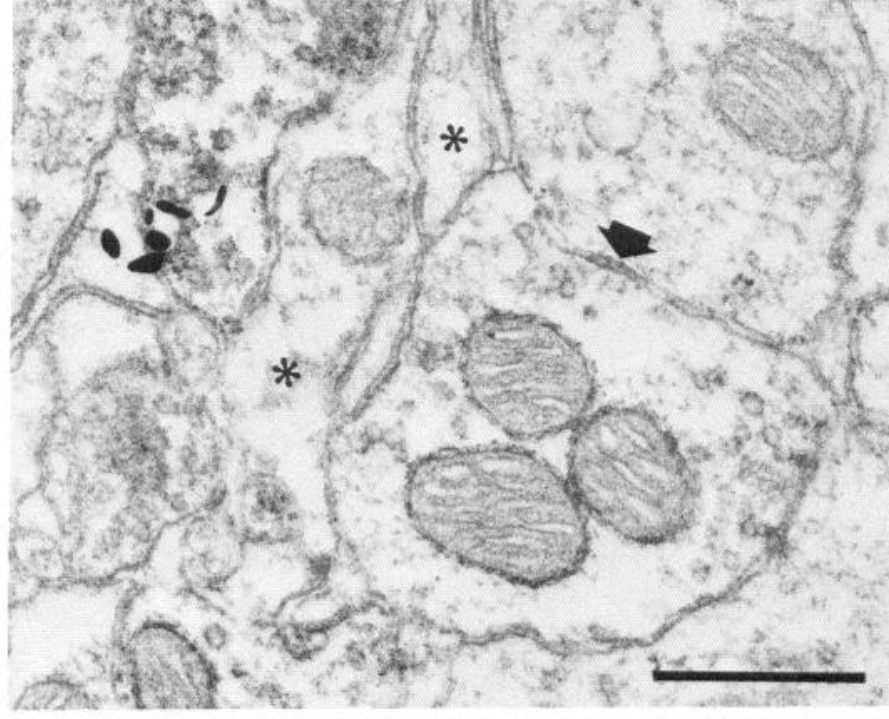

\section{UD}

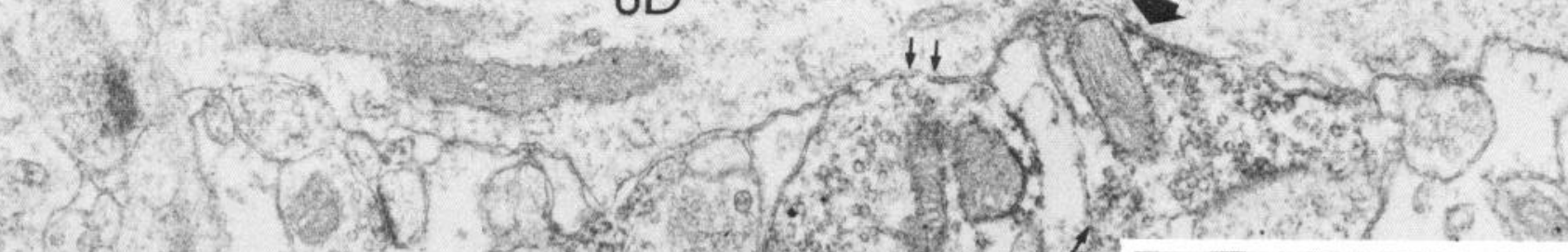

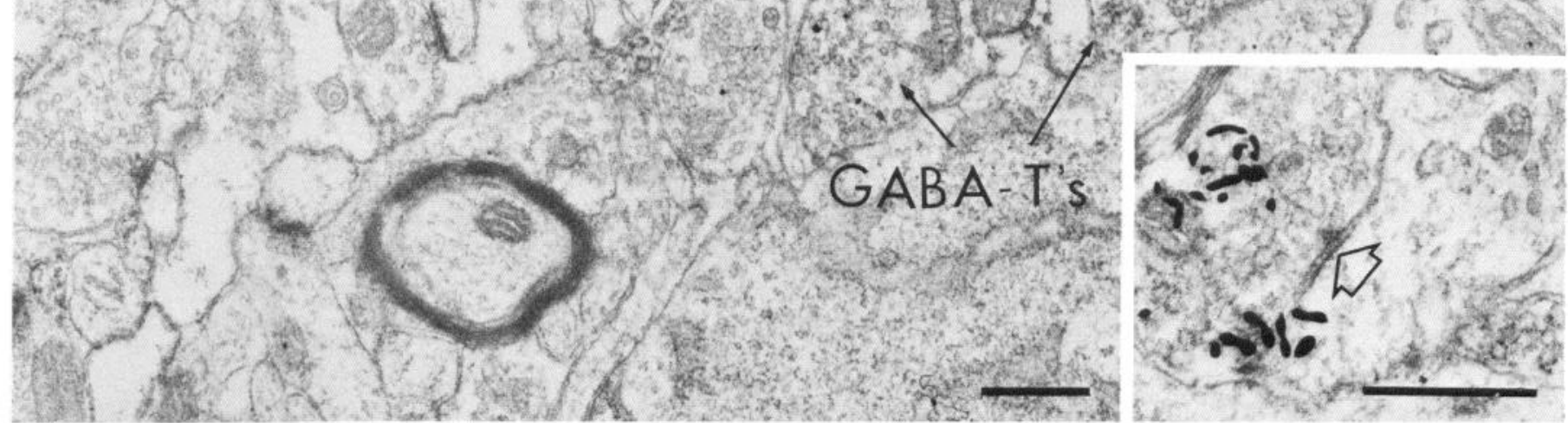


ylase (GAD), the GABA-synthesizing enzyme, in colchicine pretreated animals (Ribak et al., 1978; Amaral and Kurz, 1985; Babb et al., 1988). The majority of GABAergic neurons and processes overlapped $\mathrm{TH}$-labeled processes in the infragranular hilus of the DG and CA3 region of the hippocampus, suggesting that GABA and catecholamines could prominently interact in these regions.

By electron microscopy, the majority of GABAergic perikarya in both the DG and CA3 region were round or ovoid and contained abundant cytoplasm. The morphological features of neurons with GABA-LI were similar to those reported previously in the hippocampus using GABA or GAD antibodies in the rat (Frotscher et al., 1984; Kosaka et al., 1984; Frotscher and Zimmer, 1987; Leranth and Frotscher, 1987a, b) and human (Schlander et al., 1987). It is interesting that the ultrastructural characteristics of GABAergic neurons did not differ significantly between the hippocampus and DG since GABAergic perikarya have been divided into several subclasses based on light microscopic distribution and morphological characteristics (Seress and Ribak, 1983) and colocalization with other neurotransmitters (Somogyi et al., 1984; Kosaka et al., 1985; Kunkel and Schwartzkroin, 1988) or proteins (Kosaka et al., 1987a).

\section{Subcellular localization and sources of GABA}

The most commonly encountered type of terminal with GABALI contained numerous scvs and 1 or 2 large dcvs, a similar vesicular content to that described previously for GABAergic terminals in the hippocampus (Frotscher et al., 1984) and DG (Kosaka et al., 1984; Leranth and Frotscher, 1987a, b). The presence of dcvs amidst unlabeled smaller vesicles raises the prospect that GABA may bc colocalized in these terminals with other transmitters. Cholecystokinin, vasoactive intestinal peptide, and somatostatin are possible candidates since these substances coexist within GABAergic neurons in the hippocampal formation (Somogyi et al., 1984; Kosaka et al., 1985; Kunkel and Schwartzkroin, 1988).

Moreover, our observation that a few of the terminals with GABA-LI also contained TH-I indicates that catecholamines could be one of the coexisting transmitters in the hippocampal formation. However, neither the noradrenergic neurons in the locus coeruleus nor the dopaminergic neurons in the ventral tegmental area, which project to the hippocampus (Loy et al., 1980; Pohle et al., 1984; Haring and Davis, 1985), contain GABA (Kosaka et al., 1987b). In addition, colocalization could be attributed to uptake of GABA or its precursors. This possibility is supported by the demonstration of a carrier for GABA uptake on noradrenergic synaptosomes in hippocampus (Bonanno and Raiteri, 1987). Alternatively, the observed coexistence of GABA-LI and TH-I could be due to methological artifacts. Cross-reactivity between the respective antisera might cause erroneous detection of the 2 labels in the same terminals.
This seems unlikely in the present study since singly labeled terminals were observed immediately adjacent to terminals that were dually labeled.

There are at least 3 known sources of GABA-immunoreactive terminals in the hippocampal formation. Many GABAergic terminals originate from intrinsic neurons. The DG contains a number of GABAergic interneurons located in the infragranular hilus as well as a few GABAergic neurons in the molecular layer; the CA3 region also contains several GABAergic neurons in strata oriens, radiatum, and lacunosum-moleculare (StormMathisen et al., 1983; Gamrani et al., 1986; Sloviter and Nilaver, 1987). Additionally, GABAergic neurons in the medial septal and diagonal band nuclei (Kohler et al., 1984; Freund and Antal, 1988) and the contralateral hippocampus (Seress and Ribak, 1983; Ribak et al., 1986b) project to the hippocampal formation. Moreover, many of the GABAergic terminals in the hippocampal formation not only have morphological characteristics and synaptic associations similar to septal afferents (Chandler and Crutcher, 1983), but recently have been shown to be of septal origin (Freund and Antal, 1988).

\section{Associations of neurons with GABA-LI}

The GABA-containing neurons in the hippocampal formation received synaptic input from terminals that were either unlabeled or immunoreactive for TH or GABA.

Unlabeled terminals-GABA neurons. The majority of the terminals presynaptic to the GABA-labeled neurons in both the DG and CA3 region of the hippocampus were unlabeled and thus were unidentifiable both with respect to transmitter type and/or cellular origin. In the DG, some of these unlabeled terminals probably contain $\mathrm{ACh}$ since choline acetyltransferaseimmunoreactive terminals are known to establish symmetric synapses with GAD-labeled perikarya and dendrites (Leranth and Frotscher, 1987b). Another putative transmitter in both regions may include opioid peptides. Earlier physiological studies suggest that the opioids excite hippocampal pyramidal neurons indirectly by inhibiting neighboring inhibitory neurons (probably containing GABA) (Zieglgansberger et al., 1979; Newberry and Nicoll, 1984). In both regions, some portion of the unlabeled terminals originate from the medial septal/diagonal band nuclei (Schwerdtfeger, 1986), ipsilateral granule cells (i.e, mossy fibers) (Frotscher, 1988), or the contralateral hippocampal formation (Frotscher and Zimmer, 1983; Frotscher et al., 1984).

TH terminals-GABA neurons. The present demonstration that GABAergic perikarya and dendrites receive synapses from THlabeled terminals are in agreement with earlier dual-labeling studies using GAD and $\mathrm{TH}$ in the $\mathrm{CA} 3$ region of the hippocampus (Frotscher and Leranth, 1988). Moreover, the observations are consistent with studies of other brain regions indicating that GABAergic neurons are major targets of catecholaminergic ter-

\footnotetext{
Figure 8. Convergence of TH-containing and GABA-labeled terminals on a common dendrite in strata lucidum $(A, B)$ and radiatum $(C)$ in CA3. $A$ and $B$. Serial electron micrographs show a terminal with TH-I which forms a symmetric synapse (open arrow; more apparent in $B$ ) with an unlabeled dendrite $(U D)$ which is also postsynaptic to $2 \mathrm{GABA}$-labeled terminals (GABA-T1 and $-T 2)$ forming symmetric junctions (closed arrow). The TH-labeled terminal is also adjacent to 2 terminals with GABA-LI. Another terminal with GABA-LI $(G A B A-T 3)$ is found in the same vicinity but lacks any associations; this GABA terminal is separated from the unlabeled dendrite by glial processes (asterisks). $C$, An unlabeled dendrite $(u D)$ contacted by a terminal with TH-I (TH-T) which forms a symmetric junction (open arrow) and 2 GABA-labeled terminals ( $G A B A-T$ 's) which form a symmetric junction (closed) or is closely apposed without intervening glia (small double arrows). The dendritic spine which is contacted by the TH-labeled terminal was continuous with the shaft of the unlabeled dendrite in adjacent section (not shown). Inset at right is an adjacent section through the TH-labeled terminal. Autoradiographic exposure, 15 months. Scale bars, $0.5 \mu \mathrm{m}$.
} 
minals (Pickel et al., 1988, 1989). Junctions formed by the THlabeled terminals on GABAergic neurons were both asymmetric (Gray type I) and symmetric (Gray type II). The presence of both types of synaptic specializations are similar to our earlier electron microscopic studies of TH-labeled terminals in the hippocampal formation (Milner and Bacon, 1989). Asymmetric synapses are believed to mediate excitation. This notion is based largely on the detection of enriched populations of thickened postsynaptic densities in regions of the brain containing higher proportions of excitatory synapses; a similar analysis suggested that symmetric synapses mediate inhibition (Uchizono, 1965; Cohen et al., 1982).

The detection of excitatory (asymmetric) synapses on GABAergic interneurons agrees with the findings that norepinephrine elevates the spontaneous firing rate of theta neurons in the DG and CAl region of the hippocampus (Pang and Rose, 1987; Rose and Pang, 1989); these theta neurons exhibit many of the physiological properties associated with interneurons (Fox and Ranck, 1981; Berger et al., 1983). Moreover, in other cortical areas norepinephrine has a facilitating effect on GABA-induced or synaptically mediated inhibition (Waterhouse et al., 1982). The demonstration that the same excitatory catecholaminergic terminals synapse on pyramidal or granule cells (Milner and Bacon, 1989) as well as GABAergic neurons provides an anatomical substrate for feed-forward inhibition (Buzsaki, 1984). The occurence of inhibitory (symmetric) junctions support earlier physiological studies suggesting that norepinephrine disinhibits hippocampal pyramidal neurons by decreasing the excitability of inhibitory (probably GABAergic) interneurons (Winson and Dahl, 1985; Madison and Nicoll, 1988).

GABA terminals-GABA neurons. The detection of synaptic junctions between GABAergic neurons and GABAergic terminals is in accord with earlier reports in the hippocampal formation using GABA or GAD antibodies (Ribak et al., 1978; Gamrani et al., 1986; Misgeld and Frotscher, 1986). Recent studies combining anterograde transport of Phaseolus vulgaris with GABA immunocytochemistry indicate that the majority of GABA-containing terminals that synapse on GABAergic neurons in the hippocampus arise from the septal/diagonal band nuclei (Freund and Antal, 1988). Moreover, other dual-labeling electron microscopic studies have demonstrated that some of these postsynaptic GABAergic neurons also contain cholecystokinin (Leranth and Frotscher, 1987a). The findings of a GABAGABA interaction support the observation that hippocampal pyramidal and granule cells are depolarized by GABA (Janigro and Schwartzkroin, 1987, 1988). Some of this disinhibition of pyramidal cells by GABA occurs indirectly via nonpyramidal, inhibitory (probably GABAergic) interneurons (Misgeld and Frotscher, 1986).

\section{Associations of terminals with GABA-LI}

GABAergic terminals either alone or in conjunction with catecholaminergic terminals contact non-GABAergic hippocampal neurons. In addition, GABAergic terminals were associated with other axon terminals, some of which are GABAergic or catecholaminergic.

GABA terminals-unlabeled neurons. The identity of the unlabeled neurons postsynaptic to the GABA-labeled terminals in the hippocampal formation can be ascertained in a few cases. Consistent with previous studies (Kosaka et al., 1984; Gamrani et al., 1986), GABAergic terminals formed primarily symmetric synapses with the perikarya of pyramidal and granule cells. As previously indicated, specializations of this type are usually associated with inhibition (Uchizono, 1965; Cohen et al., 1982). The finding of symmetric junctions thus supports earlier physiological studies showing that GABA has hyperpolarizing effects when applied to hippocampal pyramidal and granule neurons (Djorup et al., 1981; Jahnsen and Laursen, 1981; Anderson et al., 1982; Newberry and Nicoll, 1984; Albertson and Joy, 1987; Rose and Pang, 1989). In the hilus of the DG, a region devoid of granule cell perikarya and dendrites, a large number of GABAergic terminals formed synapses with unlabeled perikarya and dendrites. Recent physiological studies support the concept that GABAergic terminals synapse on non-GABAergic hilar interneurons (Newberry and Nicoll, 1984). At least some of these neurons contain neuropeptide $Y$, somatostatin, cholecystokinin, and vasoactive intestinal peptide (Gall, 1984; Kohler et al., 1986; Sloviter and Nilaver, 1987). Whether the neurons containing these transmitters are targets of catecholaminergic terminals remains to be determined by future dual electron microscopic labeling techniques.

Convergence of GABA and TH terminals. A significant portion of the GABAergic terminals synapsed on the same perikarya or dendrite as the TH-labeled terminals. This observation supports physiological and pharmacological data showing that GABA potentiates submaximal norephinephrine-induced cAMP production (Karbon et al., 1984) and phospholipase C activation (Corradetti et al., 1987). It is interesting that phospholipase $\mathrm{C}$-mediated activation of protein kinase $\mathrm{C}$ may be involved in hippocampal long-term potentiation (Akers et al., 1986).

GABA terminals: other terminals. The present study demonstrates close appositions between GABA-containing and catccholamincrgic as wcll as other GABAcrgic and unlabeled terminals in the hippocampal formation. The frequency of detected appositions between GABAergic and catecholaminergic terminals could reflect presynaptic associations; such a functional relationship has been suggested previously for GABAergic and dopamine terminals in the nucleus accumbens (Pickel et al., 1988). The appositions observed between terminals are likely sites for presynaptic modulation to occur mainly because of sufficient proximity to allow for diffusion to receptive sites, and absence of intervening glial processes that have uptake sites and metabolizing enzymes for GABA and catecholamines (Schousboe et al., 1980). That GABAergic terminals may interact with noradrenergic terminals has been suggested by pharmacological studies in other brain regions and in the hippocampus showing that norepinephrine augments GABA-induced depressant actions through activation of $\beta$-adrenergic receptors (Waterhouse et al., 1982). Noradrenergic terminals also contain a carrier for GABA uptake (Bonanno and Raiteri, 1987). Moreover, GABA agonists potentiate norepinephrine-stimulated cAMP production (Karbon et al., 1984). That some of the unlabeled terminals may be cholinergic is supported by the observation that cholinergic agents can mediate pyramidal cell excitability through disruption of GABAergic transmission (Cherubini et al., 1982; Freund et al., 1988). Future dual-labeling studies similar to those employed in the present study will be necessary to demonstrate such a relationship.

\section{Functional considerations}

The results of this study provide cellular substrates in the hippocampal formation for direct synaptic modulation of (1) GABAergic neurons by both GABA and catecholamines and (2) pyramidal and granule cells, as well as interneurons, by GA- 
BAergic terminals either alone or in conjunction with catecholaminergic terminals. Moreover, the results also demonstrate that GABAergic terminals are in close apposition to other terminals, including those containing GABA or catecholamines. Interactions between GABAergic neurons and catecholaminergic terminals in the hippocampus may be important in a variety of functions including epilepsy. Numerous studies have shown that GABAergic neurons and terminals are altered either following induced epileptic seizures or in genetically epileptic animals (Ribak et al., 1986a; Peterson and Ribak, 1987). During epileptic seizure, GABA may either inhibit pyramidal or granule cell discharge through direct synaptic contacts on these neurons or disinhibit pyramidal or granule cell discharge through indirect synaptic contacts on the GABAergic interneurons. Interestingly, the increased seizure susceptibility of subcortically dencrvated hippocampus can be partially suppressed by intrahippocampal grafts of locus coeruleus (Buzsaki et al., 1988). In addition, disinhibition of pyramidal or granule cells via inhibition of GABAergic neurons could be important in long-term potentiation since such potentiation can be preferentially induced when the cells are maximally excited (Larson et al., 1986; Pavlides et al., 1988).

\section{References}

Akers, R. F., D. M. Lovinger, P. A. Colley, D. J. Linden, and A. Routtenberg (1968) Translocation of protein kinase $C$ activity may mediate hippocampal long-term potentiation. Science 231: 587-589.

Albertson, T. E., and R. M. Joy (1987) Increased inhibition in dentate gyrus granule cells following exposure to GABA-uptake blockers. Brain Res. 435: 283-292.

Alger, B. E., and R. A. Nicoll (1982a) Feed-forward dendritic inhibition in rat hippocampal pyramidal cells studied in vitro. J. Physiol. (Lond.) 328: 105-123.

Alger, B. E., and R. A. Nicoll (1982b) Pharmacological evidence for two kinds of GABA receptor on rat hippocampal pyramidal cells studied in vitro. J. Physiol. (Lond.) 328: 125-141.

Alger, B. E., C. E. Jahr, and R. A. Nicoll (1982) Electrophysiological analysis of GABA-ergic local circuit neurons in the central nervous system. Adv. Biochem. Psychol. Pharmacol. 26: 77-93.

Amaral, D. G., and J. Kurz (1985) The time of origin of cells demonstrating glutamic acid decarboxylase-like immunoreactivity in the hippocampal formation of the rat. Neurosci. Lett. 59: 33-39.

Anderson, P., R. Dingledine, L. Gjerstad, I. A. Langmoen, and A. Laursen Mosfeldt (1980) Two different responses of hippocampal pyramidal cells to application of gamma-amino butyric acid. J. Physiol. (Lond.) 305: 279-296.

Anderson, P., B. Bie, and T. Ganes (1982) Distribution of GABA sensitive areas on hippocampal pyramidal cells. Exp. Brain Res. 45: 357-363.

Babb, T. L., J. K. Pretorius, W. R. Kupfer, and W. J. Brown (1988) Distribution of glutamate-decarboxylase-immunoreactive neurons and synapses in rat and monkey hippocampus: Light and electron microscopy. J. Comp. Neurol. 278: 121-138.

Bacon, C. E., C. Abate, and T. A. Milner (1988) GABA-ergic neurons in the rat hippocampal formation: Ultrastructural characterization and synaptic relations with catecholaminergic terminals. Soc. Neurosci. Abstr. 14: 232.

Beaudet, A. (1982) High resolution autoradiography of central 5-hydroxytryptamine (5-HT) neurons. J. Histochem. Cytochem. 30:765768.

Beaudet, A., and L. Descarries (1986) Ultrastructural identification of serotonin neurons. In Ultrastructural Identification of Monoamine Neurons, J. Furness and M. Costa, eds., Wiley, London.

Berger, T. W., P. C. Rinaldi, D. J. Weisz, and R. F. Thompson (1983) Single-unit analysis of different hippocampal cell types during classical conditioning of rabbit nictitating membrane response. J. Neurophysiol. 50: 1197-1219.

Bonanno, G., and M. Raiteri (1987) A carrier for GABA uptake exists on noradrenaline nerve endings in selective rat brain areas but not on serotonin terminals. J. Neural Trans. 69: 59-70.
Buzsaki, G. (1984) Feed-forward inhibition in the hippocampal formation. Prog. Neurobiol. 22: 131-153.

Buzsaki, G., G. Ponomareff, F. Bayardo, T. Shaw, and F. H. Gage (1988) Suppression and induction of epileptic activity by neuronal grafts. Proc. Natl. Acad. Sci. USA 85: 1-4.

Chandler, J. P., and K. A. Crutcher (1983) The septohippocampal projection in the rat: An electron microscopic horseradish peroxidase study. Neuroscience 10:685-696.

Cherubini, E., C. Rovira, Y. Ben-Ari, and A. Padjen (1982) Simultaneous recording of somatic and dendritic field potentials and combined microiontophoresis in the rat Ammon's horn in situ: Effects of GABA and acetylcholine. Neurosci. Lett. 31: 19-24.

Cohen, R. S., R. K. Carlin, D. J. Grab, and P. Siekevitz (1982) Phosphoproteins in postsynaptic dendrites. Prog. Brain Res. 56: 49-76.

Corradetti, R., M. Ruggiero, V. P. Chiarugi, and G. Pepeu (1987) GABA-receptor stimulation enhances norepinephrine-induced polyphosphoinositide metabolism in rat hippocampal slices. Brain Res. 411: 196-199.

Cowan, W. M., D. I. Gottlieb, A. E. Hendrickson, J. L. Price, and T. A. Woolsey (1972) The autoradiographic demonstration of axonal connections in the central nervous system. Brain Res. 37: 21-51.

Curtis, D. R., D. Felix, and H. McLennan (1970) GABA and hippocampal inhibition. Br. J. Pharmacol. 40: 881-883.

Djorup, A., H. Jahnsen, and A. Mosfeldt-Laursen (1981) The dendritic response to GABA in the CAl of the hippocampal slice. Brain Res. 219: 196-201.

Fallon, J. H., and S. E. Loughlin (1987) Monoamine innervation of cerebral cortex and a theory of the role of monoamines in cerebral cortex and basal ganglia. In Cerbral Cortex, Vol. 6, E. G. Jones and A. Peters, eds. pp. 41-127, Plenum, New York.

Fox, S. E., and J. B. Ranck, Jr. (1981) Electrophysiological characteristics of hippocampal complex-spike and theta cells. Exp. Brain Res. 41: 399-410.

Freund, T. F., and M. Antal (1988) GABA-containing neurons in the septum control inhibitory interncurons in the hippocampus. Naturc 336: 170-173.

Freund, R. K., D. A. Jungschaffer, A. C. Collins, and J. M. Wehner (1988) Evidence for modulation of GABAergic neurotransmission by nicotine. Brain Res. 453: 215-220.

Frotscher, M. (1988) Neuronal elements in the hippocampus and their synaptic connections. In Neurotransmission in the Hippocampus, M. Frotscher, P. Kugler, U. Misgeld, and K. Zilles, eds, pp. 2-19, Springer-Verlag, Berlin.

Frotscher, M., and C. Leranth (1988) Catechominergic innervation of pyramidal and GABAergic nonpyramidal neurons in the rat hippocampus. Histochemistry $88: 313-319$.

Frotscher, M., and J. Zimmer (1983) Commissural fibers terminate on non-pyramidal neurons in the guinea pig hippocampus-a combined Golgi/EM degeneration study. Brain Res. 265: 289-293.

Frotscher, M., and J. Zimmer (1987) GABAergic nonpyramidal neurons in intracerebral transplants of the rat hippocampus and fascia dentata: A combined light and electron microscopic immunocytochemical study. J. Comp. Neurol. 259: 266-276.

Frotscher, M., C. Leranth, K. Lubbers, and W. H. Oertel (1984) Commissural afferents innervate glutamate decarboxylase immunoreactive non-pyramidal neurons in the guinea pig hippocampus. Neurosci. Lett. 46: 137-143.

Gall, C. (1984) The distribution of cholecystokinin-like immunoreactivity is the hippocampal formation of the guinea pig: Localization in the mossy fibers. Brain Res. 306: 73-83.

Gamrani, H., B. Onteniente, P. Seguela, M. Geffard, and A. Calas (1986) Gamma-aminobutyric acid immunoreactivity in the rat hippocampus. A light and electron microscopic study with anti-GABA antibodies. Brain Res. 364: 30-38.

Haring, J. H., and J. N. Davis (1985) Differential distribution of locus coeruleus projections to the hippocampal formation: Anatomical and biochemical evidence. Brain Res. 325: 366-369.

Hodgson, A. J., B. Penke, A. Erdei, I. W. Chubb, and P. Somogyi (1985) Antisera to gamma-aminobutyric acid. I. Production and characterization using a new model system. J. Histochem. Cytochem. 33: 229239.

Jahnsen, H., and A. M. Laursen (1981) The effects of benzodiazepines on the hyperpolarizing and depolarizing responses of hippocampal cells to GABA. Brain Res. 207: 214-217.

Janigro, D., and P. A. Schwartzkroin (1987) Dissociation of the IPSP 
and the response to GABA during spreading depression-like depolarization hippocampal slices. Brain Res. 404: 189-200.

Janigro, D., and P. A. Schwartzkroin (1988) Effects of GABA on CA3 pyramidal cell dendrites in rabbit hippocampal slices. Brain Res. 453: 265-274.

Joh, T. H., and M. Goldstein (1973) Isolation and characterization of multiple forms of phenylethanolamine N-methyltransferase. Mol. Pharmacol. 9: 117-129.

Joh, T. H., and M. E. Ross (1983) Preparation of catecholamine synthesizing enzymes as immunogen for immunocytochemistry. In $O x$ ford IBRO Handbook Series, Vol. 3, Immunocytochemistry, A. C. Cuello, ed., pp. 121-138, Wiley, New York.

Karbon, E. W., R. S. Duman, and S. J. Enna (1984) GABA B $_{B}$ receptors and norepinephrine-stimulated cAMP production in rat brain cortex. Brain Res. 306: 327-332.

Kohler, C., V. Chan-Palay, and J.-Y. Wu (1984) Septal neurons containing glutamic acid decarboxylase immunoreactivity project to the hippocampal region in the rat brain. Anat. Embryol. 169: 41-44.

Kohler, C., L. Eriksson, S. Davies, and V. Chan-Palay (1986) Neuropeptide $\mathrm{Y}$ innervation of the hippocampal region in the rat and monkey brain. J. Comp. Neurol. 244: 384-400.

Kosaka, T., K. Hama, and J.-Y. Wu (1984) GABAergic synaptic boutons in the granule cell layer of rat dentate gyrus. Brain Res. 293 353-359.

Kosaka, T., K. Kosaka, K. Tateishi, Y. Hamaoka, N. Yanaihara, J.-Y. $\mathrm{Wu}$, and $\mathrm{K}$. Hama (1985) GABAergic neurons containing CCK-8like and/or VIP-like immunoreactivities in the rat hippocampus and dentate gyrus. J. Comp. Neurol. 239: 420-430.

Kosaka, T., H. Katsumaru, K. Hama, J.-Y. Wu, and C. W. Heizmann (1987a) GABAergic neurons containing the $\mathrm{Ca}^{2+}$-binding protein parvalbumin in the rat hippocampus and dentate gyrus. Brain Res. 419: 119-130

Kosaka, T., K. Kosaka, Y. Hataguchi, I. Nagatsu, J.-Y. Wu, O. P. Otterson, J. Storm-Mathisen, and K. Hama (1987b) Catecholaminergic neurons containing GABA-like and/or glutamic acid decarboxylase-like immunoreactivities in various brain regions of the rat. Exp. Brain Res. 66: 191-210.

Kunkel, D. D., and A. Schwartzkroin (1988) Ultrastructural characterization and GAD colocalization of somatostatin-like immunoreactive neurons in $\mathrm{CA} 1$ of rabbit hippocampus. Synapse $2: 371-381$

Larson, L., D. Wong, and G. Lynch (1986) Patterned stimulation at the theta frequency is optimal for induction of hippocampal longterm potentiation. Brain Res. 368: 347-350.

Lauder, J. M., V. K. M. Han, P. Henderson, T. Verdoorn, and A. C. Towle (1986) Prenatal ontogeny of the GABAergic system in the rat brain: An immunocytochemical study. Neuroscience 19:465-493.

Leranth, C., and M. Frotscher (1987a) GABAergic input of cholecystokinin-immunoreactive neurons in the hilar region of the rat hippocampus. An electron microscopic double immunostaining study. Histochemistry 86: 287-290.

Leranth, C., and M. Frotscher (1987b) Cholinergic innervation of hippocampal GAD and somatostatin-immunoreactive commissural neurons. J. Comp. Neurol. 261: 33-47.

Loy, R., D. A. Koziell, J. D. Lindsey, and R. Y. Moore (1980) Noradrenergic innervation of the adult rat hippocampal formation. $J$. Comp. Neurol. 189:699-710.

Madison, D. V., and R. A. Nicoll (1988) Norepinephrine decreases synaptic inhibition in the rat hippocampus. Brain Res. 442: 131-138.

Masurovsky, E. R., and R. P. Bunge (1968) Fluoroplastic coverslips for long-term nerve tissue culture. Stain Technol. 43: 161-165.

Milner, T. A., and C. Bacon (1989) Ultrastructural localization of tyrosine hydroxylase-like immunoreactivity in the rat hippocampal formation. J. Comp. Neurol. 281: 479-495.

Milner, T. A., V. M. Pickel, C. Abate, T. H. Joh, and D. J. Reis (1988) Ultrastructural characterization of substance P-like immunoreactive neurons in the rostral ventrolateral medulla in relation to neurons containing catecholamine synthesizing enzymes. J. Comp. Neurol. 270: $427-445$

Milner, T. A., V. M. Pickel, and D. J. Reis (1989) Ultrastructural basis for interactions between central opioids and catecholamines: I. Rostral ventrolateral medulla. J. Neurosci. 9: 2114-2130.

Misgeld, U., and M. Frotscher (1986) Postsynaptic GABAergic inhibition of non-pyramidal neurons in the guinea-pig hippocampus. Neuroscience 19: 193-206.

Newberry, N. R., and R. A. Nicoll (1984) A bicuculline-resistant inhibitory post-synaptic pótential in rat hippocampal pyramidal cells in vitro. J. Physiol. (Lond.) 348: 239-254.

Ordronneau, P., P. B. M. Lindstrom, and P. Petrusz (1981) Four antibody bridge techniques. A comparision. J. Histochem. Cytochem. 29: 1397-1404.

Pang, K., and G. M. Rose (1987) Differential effects of norepinephrine on hippocampal complex-spike and O-neurons. Brain Res. 425: 146158.

Pavlides, C., Y. J. Greenstein, M. Grudman, and J. Winson (1988) Long-term potentiation in the dentate gyrus is induced preferentially on the positive phase of O-rhythm. Brain Res. 439: 383-387.

Paxinos, G., and C. Watson (1986) The Rat Brain in Stereotaxic Coordinates, 2nd ed., Academic, New York.

Peterson, G. M., and C. E. Ribak (1987) Hippocampus of the seizuresensitive gerbil is a specific site for anatomical changes in the GABAergic system. J. Comp. Neurol. 261: 405-422.

Pickel, V. M., and T. A. Milner (1987) Electron microscopy of central catecholamine systems. In Psychopharmacology: The Third Generation of Progress, H. Y. Melter, ed., pp. 49-59, Raven, New York.

Pickel, V. M., J. Chan, and T. A. Milner (1986) Autoradiographic detection of ${ }^{125}$ I-secondary antiserum: A sensitive light and electron microscopic labeling method compatible with peroxidase immunocytochemistry for dual localization of neuronal antigens. J. Histochem. Cytochem. 34: 707-718.

Pickel, V. M., A. C. Towle, T. H. Joh, and J. Chan (1988) Gammaaminobutyric acid in the medial rat nucleus accumbens: Ultrastructural localization in neurons receiving monosynaptic input from catecholaminergic afferents. J. Comp. Neurol. 272: 1-14.

Pickel, V. M., J. Chan, and T. A. Milner (1989) Cellular substrates for interactions between neurons containing phenylethanolamine $\mathrm{N}$-methyltransferase and GABA in the nuclei of the solitary tracts. J. Comp. Neurol. 286: 243-259.

Pohle, W., T. Ott, and P. Muller-Welde (1984) Identification of neurons of origin providing the dopaminergic innervation of the hippocampus. J. Hirnforsch. 25: 1-10.

Reynolds, E. S. (1963) The use of lead citrate at high pH as an electronopaque stain in electron microscopy. J. Cell Biol. 17: 208.

Ribak, C. E., J. E. Vaughn, and K. Saito (1978) Immunocytochemical localization of glutamic acid decarboxylase in neuronal somata following colchicine inhibition of axonal transport. Brain Res. 140:315332.

Ribak, C. E., C. A. Hunt, R. A. E. Bakay, and W. H. Oertel (1986a) A decreasc in the number of GABAergic somata is associated with the preferential loss of GABAergic terminals at epileptic foci. Brain Res. 363: 78-90.

Ribak, C. E., L. Seress, G. M. Peterson, K. B. Seroogy, J. H. Fallon, and L. C. Schmued (1986b) A GABAergic inhibitory component within the hippocampal commissural pathway. J. Neurosci. 6: 3492 3498.

Rose, G. M., and K. C. H. Pang (1989) Differential effect of norepinephrine upon granule cells and interneurons in the dentate gyrus. Brain Res. (in press)

Salpeter, M. M., F. A. McHenry, and E. E. Salpeter (1978) Resolution in electron microscope autoradiography. IV. Application to analysis of autoradiographs. J. Cell Biol. 76: 127-145.

Schlander, M., G. Thomalske, and M. Frotscher (1987) Fine structure of GABAergic neurons and synapses in the human dentate gyrus. Brain Res. 401: 185-189.

Schousboe, A., J. Drejer, and I. Divac (1980) Regional heterogeneity in astroglial cells. Trends Neurosci. $3: 13-14$.

Schwerdtfeger, W.K. (1986) Afferent fibers from the septum terminate on gamma-aminobutyric acid (GABA-) interneurons and granule cells in the area dentata of the rat. Experientia 42: 392-394.

Seress, L., and C. E. Ribak (1983) GABAergic cells in the dentate gyrus appear to be local circuit and projection neurons. Exp. Brain Res. 50: 173-182.

Sloviter, R. S., and G. Nilaver (1987) Immunocytochemical localization of GABA-, cholecystokinin-, vasoactive intestinal polypeptide-, and somatostatin-like immunoreactivity in the area dentate and hippocampus of the rat. J. Comp. Neurol. 256: 42-60.

Somogyi, P., A. J. Hodgson, A. D. Smith, M. G. Nunzi, A. Gorio, and J.-Y. Wu (1984) Different populations of GABAergic neurons in the visual cortex and hippocampus of the cat contain somatostatinor cholecystokinin-like immunoreactivity. J. Neurosci. 4: 2590-2603. Sternberger, L. A. (1979) Immunocytochemistry, Wiley, New York. 
Storm-Mathisen, J., A. K. Leknes, A. T. Bare, J. L. Vaaland, P. Edminson, F.-M. S. Huang, and O. P. Otterson (1983) First visualization of glutamate and GABA in neurons by immunocytochemistry. Nature 301: 517-520.

Swanson, L. B., and B. K. Hartman (1975) The central adrenergic systcm. An immunofluorescence study of the location of cell bodies and their efferent connections in the rat utilizing dopamine- $\beta$-hydroxylase as a marker. J. Comp. Neurol. 163: 467-506.

Uchizono, K. (1965) Characteristics of excitatory and inhibitory synapses in the CNS of the cat. Nature 207: 642-643.

Verney, C., M. Vaulac, B. Berger, C. Alvarez, A. Vigny, and K. B. Helle (1985) Morphological evidence for a dopaminergic terminal field in the hippocampal formation of young and adult rat. Neuroscience 14: 1039-1052.

Waterhouse, B. D., H. C. Moises, H. H. Yeh, and D. J. Woodward (1982) Norepinephrine enhancement of inhibitory synaptic mechanisms in cerebellum and cerebral cortex: Mediation by beta adrenergic receptors. J. Pharmacol. Exp. Ther. 221: 495-506.

Winson, J., and D. Dahl (1985) Action of norepinephrine in the dentate gyrus. II. Iontophoretic studies. Exp. Brain Res. 59: 497-506.

Zieglgansberger, W., E. D. French, G. R. Siggins, and F. E. Bloom (1979) Opioid peptides may excite hippocampal pyramidal neurons by inhibiting adjacent inhibitory interneurons. Science 205: 415-417. 